\title{
AU1 Capsid Engineering Overcomes Barriers Toward Adeno-Associated Viral Vector-Mediated Transduction of Endothelial Cells
}

\author{
AU2 L. Zhang, ${ }^{1,2, \dagger,}$ A. Rossi, ${ }^{3,4, \dagger}$ L. Lange, ${ }^{3,5}$ N. Meumann, ${ }^{1,3}$ U. Koitzsch, ${ }^{1,2}$ K. Christie, ${ }^{6}$ \\ M.A. Nesbit, ${ }^{6}$ C.B.T. Moore, ${ }^{6,7}$ U.T. Hacker, ${ }^{3,8}$ M. Morgan, ${ }^{3,5}$ D. Hoffmann, ${ }^{3,5}$ \\ James Zengel, ${ }^{9}$ Jan E. Carette, ${ }^{9}$ A. Schambach ${ }^{3,5,10}$ A. Salvetti, ${ }^{4}$ \\ Margarete Odenthal, ${ }^{1,2, *}$ and Hildegard Büning ${ }^{1,3,5,11, *}$ \\ AU3 ${ }^{1}$ Center for Molecular Medicine Cologne (CMMC), University of Cologne, Cologne, Germany; ${ }^{2}$ nstitute of Pathology, University Hospital of Cologne, Cologne, \\ AU4 Germany; ${ }^{3}$ Institute for Experimental Hematology and ${ }^{5}$ REBIRTH Cluster of Excellence, Hannover Medical School, Hannover, Germany; ${ }^{4}$ International Center \\ for Research in Infectiology (CIRI), INSERM U1111, CNRS UMR5308, Lyon, France; ${ }^{6}$ Biomedical Sciences Research Institute, UIster University, Ulster, \\ Northern Ireland: ${ }^{7}$ Avellino Labs USA, Menlo Park, California: ${ }^{8}$ University Cancer Center Leipzig (UCCL), Leipzig, Germany: ${ }^{9}$ Department of Microbiology and Immunology، \\ Stanford University School of Medicine, Stanford, California; ${ }^{10}$ Division of Hematology/Oncology, Boston Children's Hospital, Harvard Medical School, Boston, \\ Massachusetts; and ${ }^{11}$ German Center for Infection Research (DZIF), Partner Sites Bonn-Cologne and Hannover-Braunschweig, Braunschweig, Germany. \\ Equal contribution and shared first authorship. \\ "Current address: Ostalb-Klinikum Aalen, Medizinische Klinik II, Aalen, Germany.
}

Endothelial cells (EC) are targets in gene therapy and regenerative medicine, but they are inefficiently transduced with adeno-associated viral (AAV) vectors of various serotypes. To identify barriers hampering efficient transduction and to develop an optimized AAV variant for EC transduction, we screened an AAV serotype 2-based peptide display library on primary human macrovascular EC. Using a new highthroughput selection and monitoring protocol, we identified a capsid variant, AAV-V $\mathrm{V}_{\mathrm{EC}}$, which outperformed the parental serotype as well as first-generation targeting vectors in EC transduction. AAV vector uptake was improved, resulting in significantly higher transgene expression levels from singlestranded vector genomes detectable already few hours post-transduction. Notably, AAV-V $\mathrm{V}_{\mathrm{EC}}$ transduced not only proliferating EC but also quiescent EC, although higher particle-per-cell ratios had to be applied. Also, induced pluripotent stem cell-derived endothelial progenitor cells, a novel tool in regenerative medicine and gene therapy, were highly susceptible toward $A A V-V_{E C}$ transduction. Thus, overcoming barriers by capsid engineering significantly expands the AAV tool kit for a wide range of applications targeting EC.

Keywords: AAV vectors, endothelial cells, induced pluripotent stem cells, uncoating, uptake

\section{AU5 $-I N T R O D U C T I O N$}

ADENO-ASSOCIATED VIRAL (AAV) vectors are one of the most frequently applied gene delivery systems in preclinical research and in human clinical trials. ${ }^{1}$ Three AAV vector-based gene therapies, Glybera, Luxturna, and Zolgensma, have already received marketing authorization in the Western World. ${ }^{2}$ Further are expected to follow in the foreseeable future, because clinical benefit is reported for a wide range of monogenetic diseases. ${ }^{3}$ Firstgeneration AAV vectors are based on naturally evolved serotypes or variants of adeno-associated AU6 viruses (AAV). AAV are replication-deficient and nonpathogenic members of the Parvoviridae, with AAV serotype 2 (AAV2) as the best characterized representative and vector prototype. ${ }^{4} \mathrm{AAV}$ vectors deliver a single-stranded DNA genome into proliferating or post-mitotic cells. Cell transduction is mediated by the AAV capsid that is assembled from 60 monomers, which share the so-called common VP3 region. ${ }^{5}$ This region is identical in amino acid sequence to the major capsid protein, VP3 $(60 \mathrm{kDa})$, and contains the receptor binding motifs. In contrast, the two minor capsid proteins, VP1 $(90 \mathrm{kDa})$ and VP2 $(72 \mathrm{kDa})$, contribute distinct domains such as those required for endosomal escape and

*Correspondence: Prof Margarete Odenthal,Institute of Pathology, University Hospital Cologne, Kerpener Street62, Cologne 50937, Germany. E-mail:m.odenthal@uni-koeln.de; Dr. Hildegard Büning, Laboratory for Infection Biology and Gene Transfer, Institute of Experimental Hematology, Hannover Medical School, Carl-Neuberg Street 1, Hannover 30625, Germany. E-mail: buening.hildegard@mh-hannover.de 
nuclear delivery located within their $\mathrm{N}$-terminal extensions. ${ }^{5}$ Efficiency of in vivo gene transfer was greatly improved by using capsids of serotypes or variants others than AAV2 for packaging of recombinant AAV genomes. ${ }^{4}$ Nevertheless, still relatively high vector doses are required to obtain therapeutic effects, ${ }^{6,7}$ and distinct clinically relevant cell types such as keratinocytes or dendritic cells are refractory toward transduction. ${ }^{8,9}$

Nonsusceptibility due to lack of receptors is overcome by cell surface targeting. ${ }^{5,10}$ The same strategy is used to re-direct AAV's tropism toward a predefined cell type or a more precisely predefined cell receptor. ${ }^{5,10}$ In particular, the variable loops at the peaks of the AAV capsid and the Nterminus of the VP2 proteins accept tropismmodifying alterations without impairing capsid assembly. ${ }^{5,10}$ Lack of knowledge on peptides directing the particle toward a receptor that initiates a productive infection without interfering with capsid assembly fostered the development of viral libraries composed of capsid-engineered AAV variants that are subjected to high-throughput selection screens. Although libraries were initially based on AAV2 and displayed 7mer peptides of random sequence at capsid position $587^{11}$ or $588,{ }^{12}$ the portfolio has been expanded to other serotypes, to libraries displaying larger peptides or being composed of shuffled capsids. ${ }^{13}$ Through this powerful technology, a whole tool kit of AAV mutants with significantly improved gene delivery properties for in vitro and in vivo usage have become available. These efforts also increased our knowledge of AAV's infection biology and on cell surface molecules suited for cell entry targeting. ${ }^{5,8,14}$

Here, we focused on endothelial cells (EC), a cell type highly relevant in translational and basic research, but poorly transduced by AAV vectors with natural-occurring serotype capsids. We performed an AAV2 peptide display library screening on primary human macrovascular EC in the absence of helper virus co-infection and in the presence of high selection pressure. In addition, production of sublibraries was optimized through a novel next generation sequencing (NGS)-based amplification and cloning strategy. Thereby, AAV-V $\mathrm{V}_{\mathrm{EC}}$ was strongly selected. With $>85 \%$ of transduced cells, AAV- $\mathrm{V}_{\mathrm{EC}}$ is far more efficient than the parental serotype or firstgeneration capsid-engineered vectors at transducing EC. Using AAV- $\mathrm{V}_{\mathrm{EC}}$ and the second most enriched variant $A A V-N_{E C}$ as tools, we identified distinct steps in AAV's infection path that limit transduction of EC. Finally, we demonstrated AAV-V $V_{E C}$ 's potency in modifying induced pluripotent stem cell (iPSC)derived endothelial progenitor cells (EPC). As EPC home to damaged tissue or growing vessels,${ }^{15}$ genetic modification of such cells might be envisioned as a valuable addition to current "Trojan horse" concepts in regenerative medicine and antitumor therapy.

\section{MATERIALS AND METHODS}

\section{Cell lines and primary cells}

The human embryonic kidney cell line HEK293 (ATCC number: CRL-1573) and human cervix carcinoma cell line HeLa (ATCC number: CCL-2) were maintained in Dulbecco's modified Eagle's medium (DMEM) with GlutaMAX-I (Invitrogen, Karlsruhe, Germany) supplemented with $10 \%$ fetal bovine serum (FBS) (Invitrogen), $100 \mathrm{IU} / \mathrm{mL}$ of penicillin (Invitrogen), and $100 \mu \mathrm{g} / \mathrm{mL}$ of streptomycin (Invitrogen). AAV receptor knock-out (AAVR KO) cell lines based on HEK293 and HeLa, respectively, were generated before ${ }^{16}$ and cultivated as described earlier. Human umbilical vein EC (HUVEC) (PromoCell) was maintained in EC growth medium 2 (PromoCell) supplemented with $2 \%$ FBS, $5 \mathrm{ng} / \mathrm{mL}$ endothelial growth factor (EGF), $10 \mathrm{ng} / \mathrm{mL}$ basic fibroblast growth factor, $20 \mathrm{ng} / \mathrm{mL}$ insulin-like growth factor, $0.5 \mathrm{ng} / \mathrm{mL}$ vascular EGF (VEGF), $1 \mu \mathrm{g} / \mathrm{mL}$ ascorbic acid, $0.2 \mu \mathrm{g} / \mathrm{mL}$ hydrocortisone, 100 $\mathrm{IU} / \mathrm{mL}$ of penicillin (Invitrogen), and $100 \mu \mathrm{g} / \mathrm{mL}$ of streptomycin (Invitrogen). Throughout the experiments, only low-passage HUVEC were used (passage 4-7). All cells were maintained in a humidified incubator with $5 \% \mathrm{CO}_{2}$ at $37^{\circ} \mathrm{C}$.

\section{Cell surface expression of AAV2 receptors}

Analyses of cell surface expression of AAV2 receptors on HUVEC (low passage) and HeLa cells were performed as described. ${ }^{8,17}$ Briefly, the following primary antibodies were used in this work: mouse anti-human $\alpha \mathrm{v} \beta 5$ antibody (MAB1961; Millipore), mouse anti-human $\alpha 5 \beta 1$ antibody (MAB1999; Millipore), and mouse anti-human heparan sulfate delta antibody (USBiological). Goat anti-mouse IgG was used as secondary antibody (Southern Biotech). Cell surface expression was determined by flow cytometry (FACS Calibur; Becton Dickinson) after harvesting of cells by trypsin (HeLa; Invitrogen) or Accutase (HUVEC; Sigma) treatment. A minimum of 10,000 cells was counted for each sample.

\section{AAV peptide selection on HUVEC and $A A V$ vector production}

AAV2 peptide display library ${ }^{11}$ was produced in HEK293 cells. Phenotype and genotype of variants was coupled. Low-passage HUVEC, $24 \mathrm{~h}$ postseeding, were incubated with the AAV peptide library at a viral genome-per-cell ratio (GOI) of 1000 
on ice for $1 \mathrm{~h}$, followed by $3 \mathrm{~h}$ at $37^{\circ} \mathrm{C}$. Particles that failed to enter the cells were removed by exchanging the medium. Cells were washed and then harvested by Accutase (Sigma) treatment. After a further washing step, total DNA was isolated (DNeasy Blood and Tissue kit; Qiagen). Amounts of particles that successfully entered cells were quantified by quantitative polymerase chain reaction (qPCR) using cap gene-specific primers. Cap sequences neighboring the insertion site were amplified by

- AU7 PCR and sub-cloned into pLG backbone with CLO

ST1 primers (Supplementary Table S1). The sub-library was produced as described earlier. Meanwhile, one third of viral DNA was PCR amplified with NGS primers for sequencing. To raise the selection pressure, GOI was reduced in subsequent selection rounds (GOI 100 for 2 nd and GOI 10 for 3rd round). The 3rd round of AAV peptide display selection was repeated. Cells were harvested, and DNA was isolated from cell nuclei after subcellular fractionation (Subcellular Protein Fractionation Kit for Tissues; Thermo Fischer Scientific).

NGS data obtained from that selection (SubNuc) were compared with NGS data from round 1 to 3 of whole cell selection (Sub-Lib1-Sub-Lib3). Purity of fraction at the protein level was assayed

AU9 by western blot analysis (Supplementary Fig. S2) using antibodies against AKT (cytosol; Cell Signaling Technologies, Inc.) and Lamin B (nucleus; Santa Cruz Biotechnology, Inc.).

Peptide sequences of the most prominent two variants were ordered as oligonucleotides and cloned into pRC'99 by using MluI/AscI sites ${ }^{18}$ generating pRC'99- $\mathrm{V}_{\mathrm{EC}}$ and pRC'99- $\mathrm{N}_{\mathrm{EC}}$, respectively. For production of AAV vectors, HEK293 cells were cotransfected with a total of $37.5 \mu \mathrm{g}$ of $\mathrm{pRC},{ }^{19}$ pRC'99-V $\mathrm{V}_{\mathrm{EC}}$, pRC'99-N $\mathrm{EC}$, pRC'99-SIG ${ }^{18}$ or pRC'99NDVRAYS, pscAAV/EGFP ${ }^{20}$ or $\mathrm{pGFP}^{21}$ and pXX6. ${ }^{22}$ Of note, the AAV helper plasmid pRC'99NDVRAYS was cloned as described in Nicklin et $a l .{ }^{18}$ by using the peptide sequence reported by Muller et $a l .{ }^{12}$ Cells were harvested $48 \mathrm{~h}$ posttransfection, cell pellet was lysed, and vectors were purified by iodixanol step gradient centrifugation. ${ }^{20}$ Genomic particle titers were determined by qPCR (LightCycler System; Roche Diagnostics) using green fluorescent protein (GFP)-specific primers, ${ }^{20}$ whereas capsid titers were determined by A20ELISA (Progen).

\section{Quantification of cell entry and nuclear entry efficiency}

For determining vector uptake, HUVEC were incubated with the indicated GOI for $30 \mathrm{~min}$ on ice, followed by a shift to $37^{\circ} \mathrm{C}$ and $5 \% \mathrm{CO}_{2}$. Cells were harvested at the indicated time points posttransduction (p.t.) by Accutase or trypsin treatment and washing steps as described earlier. Total DNA was isolated (DNeasy Tissue Kit; Qiagen), and vector genomes were quantified by qPCR (Roche Diagnostics) using GFP-specific and plasminogen activator (PLAT)-specific primers. ${ }^{23}$ After confirmation of target specificity by melting curve analyses, GFP values were normalized to PLAT levels by using the LightCycler 480 software 1.5 (Roche Diagnostics). For determining nuclear entry efficiency, subcellular fractionation was performed as described earlier. The nuclear fractions were subjected to DNA isolation and analyzed as described earlier.

\section{Cell binding assay}

For determining the cell surface binding, HUVEC were seeded $24 \mathrm{~h}$ before transduction in 12 well plates with a density of $2.5 \times 10^{5}$ cells per well. Cell number per well was determined before transduction and used to calculate the GOI. To synchronize cell transduction, cells were incubated on ice for $10 \mathrm{~min}$, followed by a washing step with phosphate-buffered saline (PBS) (cold). Cold fresh media were added to cells, which were maintained on ice for a further $20 \mathrm{~min}$. Then, indicated vectors $\left(\right.$ GOI $\left.5 \times 10^{3}\right)$ diluted in cold media were added to the cells. After a further 1-h incubation on ice, one sample for each vector-corresponding to the time point $0 \mathrm{~h}$ p.t.- was washed with PBS intensively (two times), harvested by trypsin treatment, and finally stored at $-80^{\circ} \mathrm{C}$. The remaining samples were incubated at $37^{\circ} \mathrm{C}$ until harvesting $(4,6$, and 24 h p.t.). Again, supernatant was removed, and cells were washed with PBS. Trypsin treatment followed by two washing steps of the cell pellet was performed to remove membrane-bound particles. A part of the " $24 \mathrm{~h}$ samples" was analyzed by FACS (CytoFLEX platform; Beckman Counter). All samples were then subjected to DNA isolation and qPCR analyses as described earlier.

\section{Transduction of HUVEC, AAVR KO, and iPSC-derived EPC}

For determining transduction efficiency, HUVEC, HEK293-AAVR-KO, HEK293, HeLa-AAVR$\mathrm{KO}$, and HeLa cells, respectively, were incubated with indicated vectors and GOI for $24 \mathrm{~h}$ followed by flow cytometric analyses (FACS Calibur; Becton Dickinson). Background fluorescence was set to $1 \%$ by using nontreated cells as reference. For transduction of quiescent EC, HUVEC were cultivated until they reached a confluency of $\sim 90 \%$, followed by incubation for another $24 \mathrm{~h}$ at $37^{\circ} \mathrm{C}$ and $5 \% \mathrm{CO}_{2}$ with EC Basal Medium (PromoCell), which was 
devoid of all supplements and FBS. Finally, the status of quiescence was confirmed by staining with 5-ethynyl-2'-deoxyuridine (EdU) according to the manufacturer's instruction (Invitrogen).

For transduction of iPSC-derived EPC (iEC), iPSC clone $\mathrm{H} 2 \mathrm{E} 6 \mathrm{C}^{24}$ was differentiated into EC lineages by using a modified protocol based on a previously described procedure. ${ }^{25}$ Briefly, iPSC were cultured

AU10 as monolayers in MEF-conditioned medium on Geltrex (Thermo Fischer Scientific) and mesodermal induction was initiated by the GSK3-inhibitor CHIR (Axon Medchem). Cells were further differentiated into EPC in VEGF (Peprotech) containing medium for 6 days. On day 7 of differentiation, EC were harvested by using StemPro Accutase (Thermo Fischer Scientific) and seeded on a gelatin (SigmaAldrich)-coated plate in EGM2 medium (Lonza). Transduction was performed at indicated days of differentiation with indicated vectors in EGM2 medium. GFP expression was analyzed $48 \mathrm{~h}$ p.t. by flow cytometry (FACS Calibur; Becton Dickinson).

\section{Heparin competition assay}

Overall, $2.5 \times 10^{4}$ HUVEC per well were seeded $24 \mathrm{~h}$ before transduction. This cell number was used for calculation of GOI. Cells were incubated for 10 min on ice; after a washing step (cold PBS), cold fresh media were added to the cells, which were maintained on ice for a minimum of $20 \mathrm{~min}$. Viral vectors were diluted in heparin containing medium and added to the cells (final concentration indicated

AU11 in figure). One hour post-vector application, samples were shifted to $37^{\circ} \mathrm{C}$ for $24 \mathrm{~h}$. Percentage of transgene-expressing cells was determined by using Cytometry (CytoFLEX platform; Beckman Counter).

\section{Heparin affinity chromatography}

A HiTrap heparin affinity column (Amersham) was equilibrated with $1 \times \mathrm{PBS} / 1 \mathrm{mM} \mathrm{MgCl} / 2.5 \mathrm{mM}$ $\mathrm{KCl}$. Gradient-purified AAV vector preparation diluted in $1 \times \mathrm{PBS} / 1 \mathrm{mM} \mathrm{MgCl}_{2} / 2.5 \mathrm{mM} \mathrm{KCl}$ was loaded. Flow-through and $4 \times 5 \mathrm{~mL}$ wash fraction $(1 \times \mathrm{PBS} / 1 \mathrm{mM} \mathrm{MgCl} / 2.5 \mathrm{mM} \mathrm{KCl})$ were collected. Elution was performed with increasing ionic strength $\left(1 \times \mathrm{PBS} / 1 \times \mathrm{mM} \mathrm{MgCl}_{2} / 2.5 \times \mathrm{mM} \mathrm{KCl}\right.$ plus $100 \mathrm{mM} \mathrm{NaCl}$ up to $1 \times \mathrm{PBS} / 1 \mathrm{mM} \mathrm{MgCl}_{2} / 2.5 \mathrm{mM}$ $\mathrm{KCl}$ plus $1 \mathrm{M} \mathrm{NaCl}$. For each step, five column volumes were collected. Samples were analyzed by qPCR using transgene (GFP)-specific primers.

\section{Capsid thermal stability assay}

We performed the capsid thermal stability assay as described. ${ }^{26}$ Briefly, wells of qPCR plates were loaded with $5 \times 10^{8}$ vector genome containing particles of indicated AAV vector preparations diluted in PBS. The temperature gradient was generated by a LightCycler ${ }^{\circledR} 96$ System (Roche Life Science). Then, PBS was used to dilute samples. Samples were transferred to a nitrocellulose membrane by using a vacuum blotter at native dot blot conditions. Membrane was blocked and then incubated with primary (A20, or B1; Progen) and then secondary (peroxidase-conjugated antibodies) antibodies. Finally, membranes were incubated with an enhanced chemiluminescence reagent (West Dura; Pierce) and analyzed by autoradiography film exposure or FusionFX device (Peqlab).

\section{Statistical analysis}

Statistical analyses and significance tests were performed by applying unpaired, two-tailed $t$-tests. $p$-values of $<0.05$ were considered significant.

\section{RESULTS}

\section{Insufficient levels of heparan sulfate} proteoglycan may limit EC transduction

At a vector GOI with which more than $80 \%$ of HeLa cells were transduced with a single-stranded AAV2 vector encoding for enhanced GFP, only $18.0 \pm 4.95 \%$ of HUVEC expressed the reporter gene (Fig. 1A). This result is consistent with earlier $\varangle \mathrm{F} 1$
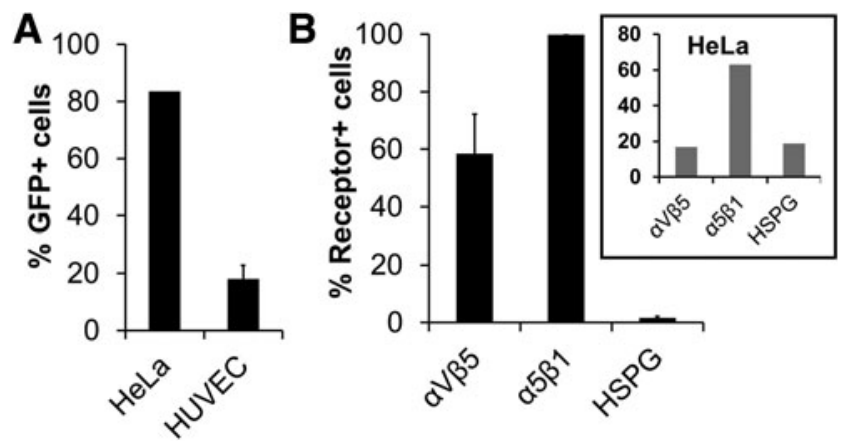

Figure 1. Vascular endothelial cells are poor targets for AAV2 transduction. (A) Transduction efficiency on low-passage HUVEC. HeLa cells or low-passage HUVEC were incubated with AAV2 vectors encoding for enhanced GFP controlled by the human CMV promoter in a single-stranded vector genome conformation at a $\mathrm{GOI}$ of 10,000 . Percentage of GFPexpressing cells was determined by flow cytometry $24 \mathrm{~h}$ p.t. Shown are mean values of two (HeLa) or three (HUVEC) independent experiments. Error bars indicate SD. (B) Expression of AAV2 receptors. Low-passage HUVEC were stained with mouse anti-human heparan sulfate delta, $\alpha 5 \beta 1$ and $\alpha \mathrm{v} \beta 5$ integrin antibodies, respectively, followed by a phycoerythrinlabeled IgG secondary antibody. Percentage of cells positive for HSPG or indicated integrins was quantified by flow cytometry. Shown are mean values of three independent experiments. Error bars indicate SD. Insert. same stainings were performed on HeLa cells. Shown is the mean of two independent experiments. AAV2, adeno-associated viral serotype 2; CMV, cytomegalovirus; GFP, green fluorescent protein; GOI, genome-per-cell ratio; HSPG, heparan sulfate proteoglycan; HUVEC, human umbilical vein endothelial cells; p.t., post-transduction; SD, standard deviation. 
reports showing that AAV2 and other AAV serotypes are inefficient in EC transduction. ${ }^{12,18,27,28}$

Low permissiveness is frequently associated with inefficient provision of receptors; therefore, HUVEC were analyzed for the presence of AAV2's primary receptor, heparan sulfate proteoglycan (HSPG) ${ }^{29}$ and the internalization receptors $\alpha \mathrm{v} \beta 5$ and $\alpha 5 \beta 1$ integrin, ${ }^{30,31}$ respectively. As shown in Fig. 1B, HUVEC clearly stained positive for $\alpha \mathrm{v} \beta 5$ and $\alpha 5 \beta 1$, whereas HSPG was barely detectable. In contrast, HeLa cells showed considerable expression of both, HSPG and the two integrins (Fig. 1B, insert).

Given the dependency of AAV2 on HSPG for subsequent steps in cell transduction, such as priming the capsid for co-receptor binding, endosomal escape, and uncoating, ${ }^{32}$ it is reasonable to postulate that the low HSPG expression level negatively impacts AAV2 transduction efficiency.

\section{AAV peptide selection of AAV capsid variants on HUVEC}

To develop an AAV variant that overcomes this pre- and maybe further post-entry barriers, an $\mathrm{AAV}$ peptide display screening was performed. The library was based on AAV2, displayed random
7 mer peptides at position 587 , and was screened on HUVEC by applying the following criteria: (1) Selections were performed in the absence of helper virus co-infection to avoid any assistance in cell infection caused either directly by helper virus proteins or indirectly by changing the intracellular milieu, (2) time of library exposure was limited to three hours, and (3) selection pressure was constantly increased by decreasing the GOI to select for highly infectious variants.

In total, three rounds of selection were performed on low-passage HUVEC. Each time, viral genomes were isolated from whole cell extract. Genomes served as the template for sub-library production by using a novel NGS-based protocol (Supplementary 4 SF1 Fig. S1). In parallel, NGS was performed to monitor the selection procedure (Fig. 2). Cluster analysis $4 \mathrm{~F} 2$ revealed a strong selection of two viral variants, AAV-VSSSTPR and AAV-NNPLPQR (Fig. 2A). Both variants were recovered with a frequency of $0.03 \%$ from the 1st round of selection. Frequency of occurrence increased to $0.42 \%$ (VSSSTPR) and $0.88 \%$ (NNPLPQR) in the 2nd and finally to $2.93 \%$ (VSSSTPR) and $3.23 \%$ (NNPLPQR) in the 3rd round of selection.
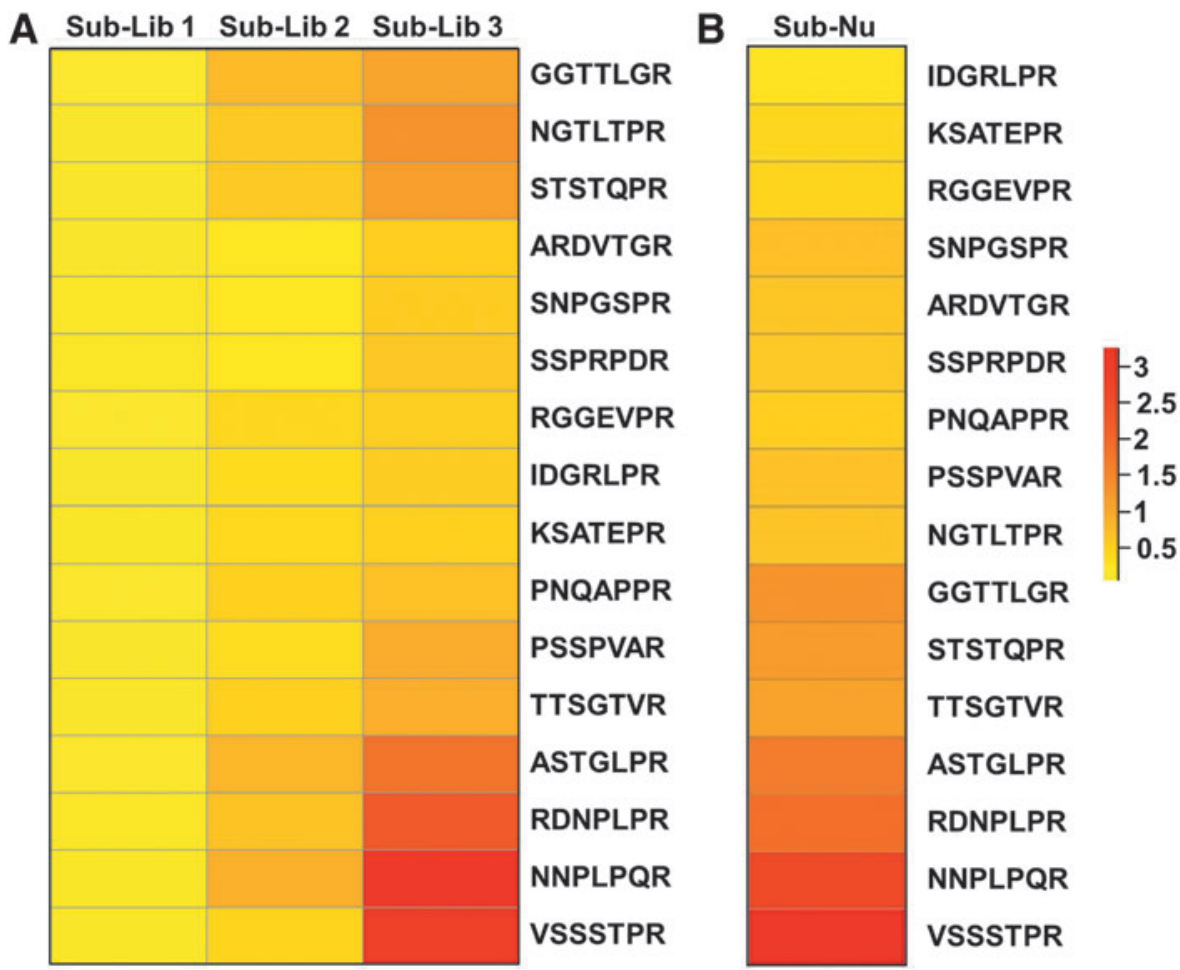

Figure 2. Heatmap of variants determined by NGS. (A) Sequences recovered from whole cell lysates. After each round of selection, viral genomes isolated from library-infected HUVEC were subjected to NGS. Between 18,000 (sub-library 2) and 26,000 (sub-library 1) reads were performed. Results of selection round 1 (GOI 1000), round 2 (GOI 100), and round $3(\mathrm{GOI} 10)$ are shown. Color code represents the frequency of occurrence for each clone according to the R-package heatmap (Pretty Heatmaps by Raivo Kolde). (B) Sequences recovered from nuclear fraction. Sub-library 2 was used to infect HUVEC (GOI 10), followed by isolation of the nuclear fraction (Supplementary Fig. S2) and NGS of isolated viral genomes. NGS, next generation sequencing. 
Isolation of viral variants from whole cell lysate indicates successful viral cell entry; however, it does not indicate the ability of the mutants to reach the nucleus. Consequently, the 3rd round of selection was repeated. When cells were harvested this time, nuclei were isolated (Supplementary Fig. S2) and then subjected to NGS sequencing to identify viral variants that accumulated in this cell compartment (Fig. 2B). Comparing the sequence pattern of this selection with those obtained for the whole cell lysate suggests that indeed not all variants reached the nuclear area with the same efficiency (Fig. 2A vs. B). VSSSTPR and NNPLPQR, however, remained the most frequently selected variants, although the ranking changed (3.27\% for VSSSTPR vs. $2.62 \%$ for NNPLPQR). Analyzing genomes encoding for VSSSTPR and NNPLPQR, respectively, revealed at least three independent selection events, that is, selection of three independent clones

T1 for each of the two peptide motifs (Table 1).

\section{AAV targeting vectors transduce HUVEC with higher efficiency}

To characterize the tropism of AAV-VSSSTPR and AAV-NNPLPQR, variants were produced as vectors $\left(A A V-V_{E C}\right.$ and $A A V-N_{E C}$ ) displaying the respective peptide at position 587 in each of the 60 capsid subunits. Vectors again delivered GFP as reporter gene in a single-stranded vector genome conformation. AAV2 was prepared in parallel, to act as a control. The parental serotype and the newly developed variants showed a comparable packaging efficiency, revealing that ligand insertion did not interfere with vector production or with vector ge-

ST2 nome packaging (Supplementary Table S2).

Vectors were then tested on low-passage HU-

F3 VEC (Fig. 3). Of the two mutants, AAV-V $\mathrm{EC}_{\mathrm{EC}}$ showed the highest transduction efficiency at all vector GOIs. At the lowest GOI (GOI 3600), AAV-V $\mathrm{V}_{\mathrm{EC}}$ transduced $28.1 \pm 6.8 \%$ of HUVEC cells. Transduction rate increased to up to $68.2 \pm 6.9 \%$ at a GOI of 30,000 , representing an up to 2.7 -fold increase in

Table 1. Viral genomes encoding selected capsid-modified adeno-associated viral variants

\begin{tabular}{llr}
\hline Motif & Sequence & $\%$ \\
\hline VSSSTPR & GTG AGC AGC TCG ACC CCG AGG & 99.54 \\
& GTG AGC AGC TCA ACC CCG AGG & 0.31 \\
NNPLPOR & GTA AGC AGC TCG ACC CCG AGG & 0.15 \\
& AAC AAC CCG CTC CCG CAG CGG & 98.42 \\
& AAC AAC CCG CTC CCA CAG CGG & 1.38 \\
& AAC AAC CCG CTC CCG CAA CGG & 0.20 \\
\hline
\end{tabular}

Next generation sequencing revealed selection of the same peptide motif by different viral clones. The percentage represents the portion of the individual sequence coding for the corresponding motif. transduction efficiency over AAV2. In contrast, although selected with a comparable efficiency as $\mathrm{AAV}-\mathrm{V}_{\mathrm{EC}}, \mathrm{AAV}-\mathrm{N}_{\mathrm{EC}}$ was significantly less efficient in transducing HUVEC. However, AAV- $\mathrm{N}_{\mathrm{EC}}$ demonstrated a significantly improved EC transduction rate as compared with AAV2 at the highest vector dose tested.

Particularly in primary cells and in vivo, conversion of single-stranded AAV (ssAAV) vector genomes into a transcriptionally active doublestranded conformation has been identified as a post-entry barrier that can be overcome by usage of a self-complementary genome conformation. ${ }^{33}$ To evaluate whether EC transduction efficiency can be further improved by switching to a selfcomplementary genome design, we performed a sideby-side comparison of conventional single-stranded and self-complementary vector genomes delivered by AAV-V $\mathrm{V}_{\mathrm{EC}}$, our best performing variant (Fig. 4). $4 \mathrm{~F} 4$ Especially at lower GOI, self-complementary AAV (scAAV)- $V_{E C}$ showed a higher transduction efficiency compared with ssAAV-V $V_{\mathrm{EC}}$. Of note, scAAV$\mathrm{V}_{\mathrm{EC}}$ at a GOI of 2500 was already sufficient to achieve a transduction efficiency of $50 \%$.

\section{AAV- $V_{E C}$ is superior to previously developed capsid variants}

AAV targeting vectors for EC transduction were previously developed. ${ }^{12,18,28,34-39}$ The first example—already described in 2001-is AAV-SIG,

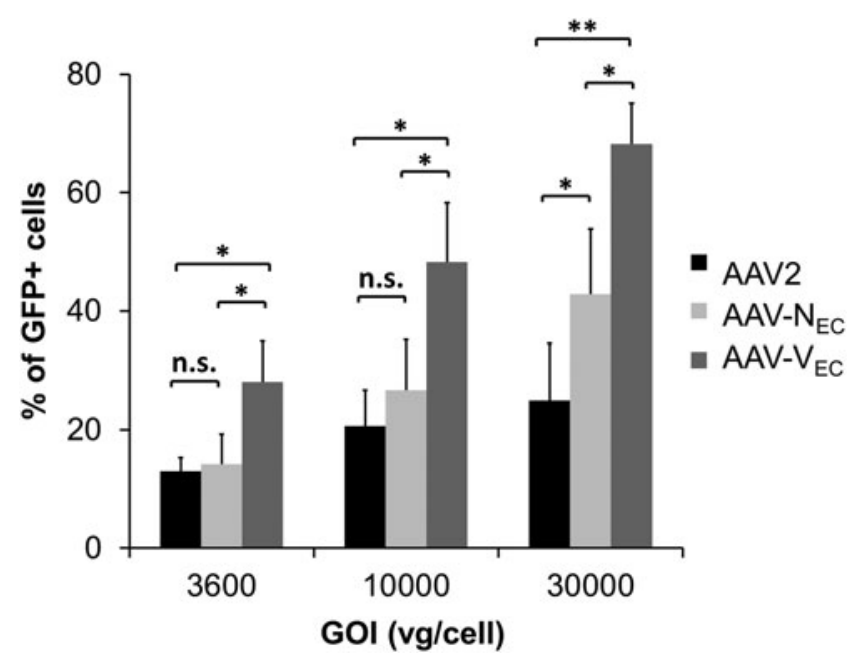

Figure 3. Transduction efficiency of AAV variants compared with AAV2. Low-passage HUVEC were incubated with a serial dilution of AAV2 (black), AAV-N $\mathrm{N}_{\mathrm{EC}}$ (light gray), or AAV-V $\mathrm{V}_{\mathrm{EC}}$ (dark gray). Vectors encode for GFP controlled by CMV promoter in a single-stranded vector genome conformation. Numbers on $X$-axis indicate genome (vg)-per-cell ratio. The percentage of transgene (GFP)-expressing cells was determined by flow cytometry $24 \mathrm{~h}$ p.t. Experiments were performed three times independently. Error bars indicate SD. ${ }^{* *} p<0.001 ;{ }^{*} p<0.05$. n.s., nonsignificant; vg, vector genome. 
A
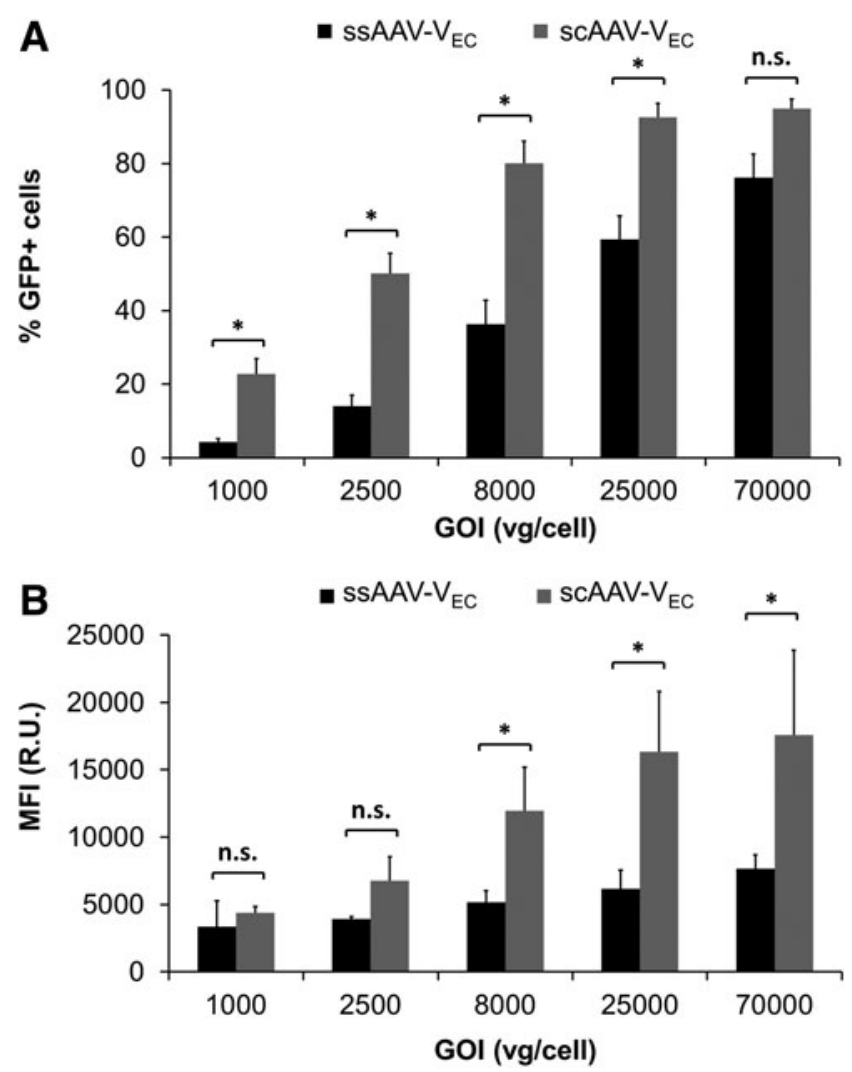

Figure 4. Self-complementary vector genome conformation improves transduction efficiency. Low-passage HUVEC were incubated with a serial dilution of $\mathrm{AAV}-\mathrm{V}_{\mathrm{EC}}$ delivering a single-stranded (ss; black) or selfcomplementary (sc, dark gray) vector genome encoding for GFP controlled by a CMV promoter. Numbers on $X$-axis indicate GOI. Percentage of GFPexpressing cells (A) and mean fluorescence intensity (B) were determined $24 \mathrm{~h}$ p.t. by flow cytometry. Shown are results of three independent experiments \pm SD. ${ }^{*} p<0.05$.

AU12 which displays the SIGYPLP peptide at position $587 .{ }^{18}$ Direct comparison of AAV-SIG with AAV-V by incubating low-passage HUVEC at a GOI of 10,000 , followed by quantification of GFP-expressing cells $24 \mathrm{~h}$ later, revealed $\mathrm{AAV}-\mathrm{V}_{\mathrm{EC}}$ to have a 13 -fold

F5 greater transduction efficiency (Fig. 5A).

Although the SIGYPLP peptide was selected on HUVEC, the same cell type as AAV-V $\mathrm{V}_{\mathrm{EC}}$, but by phage display and not by AAV peptide display, Muller et al. performed the first AAV2 peptide display on EC and identified AAV-NDVRAVS as an efficient variant for transducing human coronary artery EC. ${ }^{12}$ Comparison of AAV-NDVRAVS with $\mathrm{AAV}-\mathrm{V}_{\mathrm{EC}}$ again revealed the superiority of AAV$\mathrm{V}_{\mathrm{EC}}$, which transduced HUVEC with a fourfold higher efficiency (Fig. 5B).

\section{Barriers toward AAV2-mediated transduction of vascular EC}

AAV peptide display selects from a pool of capsid variants those that comply best with the selection
A
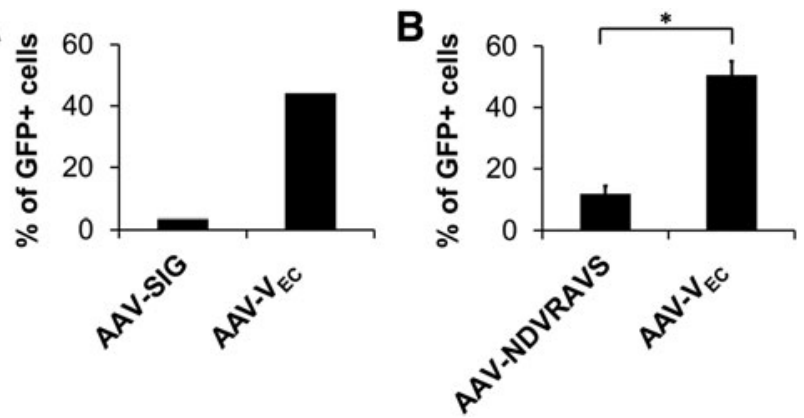

Figure 5. Improved transduction efficiency compared with previously developed capsid-engineered AAV vectors. Low-passage HUVEC were incubated with indicated capsid-modified vectors encoding for GFP in a single-stranded vector genome conformation at $\mathrm{GOI}$ of 10,000 . The percentage of GFP-expressing cells was determined by flow cytometry $24 \mathrm{~h}$ p.t. (A) Mean value of two independent experiments; (B) mean of three independent experiments \pm SD. ${ }^{*} p<0.05$.

criteria. Our results argue that, in particular, not only $\mathrm{AAV}-\mathrm{V}_{\mathrm{EC}}$ but also AAV-N $\mathrm{N}_{\mathrm{EC}}$ possess features that are beneficial for EC transduction that are not only superior to other variants in our library but also superior to AAV2 (Fig. 3). Based on this, we characterized the vector-EC interaction in more detail.

The kinetics and efficiency of cell entry as an indicator of successful uptake were determined by quantifying intracellular vector genomes at indicated time points by qPCR (Fig. 6). Highest vector $<$ F6
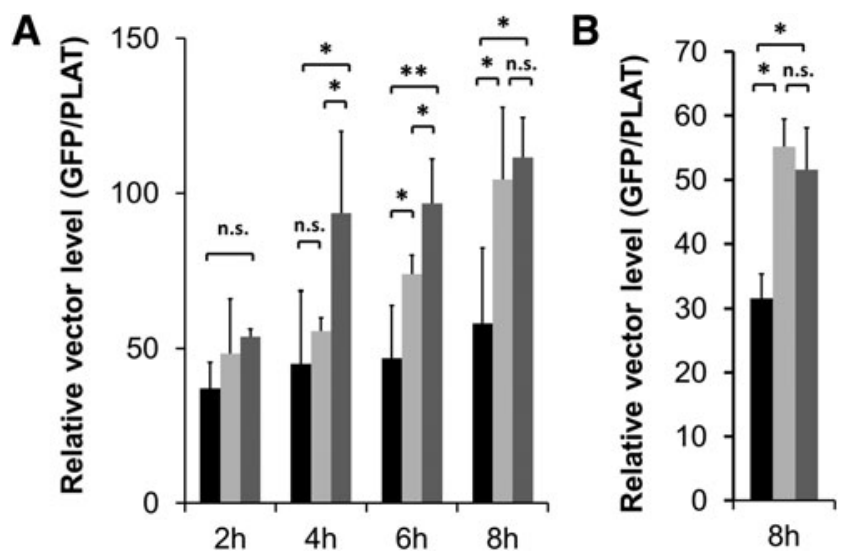

Figure 6. AAV targeting vectors differ in pre-entry and intracellular steps. Low-passage HUVEC were incubated with AAV2 (black), AAV-N $\mathrm{N}_{\mathrm{EC}}$ (light gray), or AAV-V $\mathrm{V}_{\mathrm{EC}}$ (dark gray), at a $\mathrm{GO}$ of 10,000 . Cells were harvested at the indicated time points (p.t.), and unbound and membrane-bound particles were removed. (A) Intracellular particles in whole cell lysate: total DNA was isolated, and number of intracellular vector genomes was quantified by qPCR using GFP- and PLAT-specific primers. Values obtained for vector genomes were normalized to values for PLAT. Shown are results of three independent experiments \pm SD. (B) Particles in nuclear fraction: Vectortreated cells were subjected to cell compartmentalization $8 \mathrm{~h}$ p.t. Total DNA was isolated from the nuclear fraction. Vector genomes were quantified by qPCR using GFP-specific primers and normalized to PLAT. Shown are results of three independent experiments $\pm S D$. ${ }^{* *} p<0.001 ;{ }^{*} p<0.05$. PLAT, plasminogen activator; qPCR, quantitative polymerase chain reaction. 
copy numbers (VCN) were detected for $\mathrm{AAV}-\mathrm{V}_{\mathrm{EC}}$ at all time points. $\mathrm{AAV}-\mathrm{V}_{\mathrm{EC}}$ and the parental serotype AAV2 reached a plateau at $4 \mathrm{~h}$ p.t., whereas VCN continuously increased with time in the case of AAV-N $\mathrm{NC}_{\mathrm{EC}}$, reaching a VCN comparable to AAV-V $\mathrm{V}_{\mathrm{EC}}$ at $8 \mathrm{~h}$ p.t. To discriminate whether improved binding or internalization is responsible for the improved entry efficiency, we measured cell binding in the cold and again intracellular vector par-

$\mathrm{SF} 3$ ticles on shifting samples to $37^{\circ} \mathrm{C}$ (Supplementary Fig. S3). Although AAV-V $\mathrm{EC}_{\mathrm{E}}$ and AAV2 did not differ in cell binding, we again observed significantly higher intracellular $\mathrm{VCN}$ for $\mathrm{AAV}-\mathrm{V}_{\mathrm{EC}}$ compared with AAV2. Overall, the results indicate that $\mathrm{AAV}-\mathrm{V}_{\mathrm{EC}}$ is internalized into HUVEC with a significantly higher efficiency than AAV2.

Determination of intranuclear vector genomes at $8 \mathrm{~h}$ p.t. by isolating cell nuclei of vector-treated cells followed by qPCR measurements revealed that $\mathrm{AAV}-\mathrm{V}_{\mathrm{EC}}$ and $\mathrm{AAV}-\mathrm{N}_{\mathrm{EC}}$ showed a comparable VCN, which again was significantly higher than the VCN of AAV2 (Fig. 6). Measuring VCN does not allow to discriminate between vector genomes that have been released from the capsid (uncoating) and those that are still contained in the capsid and are thus not available for transcription. As an indirect measure for uncoating efficiency, we therefore determined the onset and level of transgene expression. Interestingly, our two targeting vectors that reached the nuclear area with comparable efficiency differed with regard to the onset of transcriptional activity and transgene expression level, arguing for an improved uncoating efficiency of

F7 AAV-V $\mathrm{E}_{\mathrm{EC}}$ compared with AAV-N $\mathrm{N}_{\mathrm{EC}}$ (Fig. 7). Not only AAV- $\mathrm{N}_{\mathrm{EC}}$ but also AAV2 demonstrated a significantly later onset of transcriptional activity (Fig. 7A), a difference confirmed by flow cytometry (Fig. 7B). However, in case of AAV2, it remains to be shown whether the lower transcriptional activity is, indeed, due to a lower uncoating efficiency or caused by the lower of number of particles since significantly fewer AAV2 particles were internalized and accumulated in the nuclear area. An argument for the former, however, is the lower (thermal) stability of $\mathrm{AAV}-\mathrm{V}_{\mathrm{EC}}$ compared with SF4 AAV2 (Supplementary Fig. S4).

\section{AAV-V $V_{E C}$ binds heparin, a soluble analogue of HSPG}

Insertion of peptides in position 587 of the AAV capsid proteins destroys the HSPG-binding motif of wild-type AAV $2,{ }^{40}$ which hampers the natural infection process. This is an advantage for cell surface targeting approaches aiming at re-directing AAV's tropism. ${ }^{40}$ However, on insertion of overall positively charged peptides or of peptides that form an HSPGbinding motif, targeting vectors regain the ability to bind to HSPG although affinity might differ. ${ }^{41,42}$

Interestingly, our selection on HUVEC, which were performed without any selection pressure regarding specificity of cell transduction, yielded exclusive variants that present an arginine $(R)$ residue at position 7 of the peptide insertion (Fig. 2). This arginine might form together with the two linker amino acids (AA in our case) and R588 of the wild-type AAV2 sequence a heparin binding motif $(\mathrm{RXXR})^{41,42}$ that contributes to cell trans- $4 \mathrm{AU} 13$ duction. To test whether AAV- $\mathrm{V}_{\mathrm{EC}}$ is able to bind to HSPG, we performed a heparin affinity chromatography and eluted viral particles with increasing salt concentration (Fig. 8A). For comparison, AAV2 4 F8 was subjected to the same test. AAV2 was eluted as a single peak when adding $300 \mathrm{mM} \mathrm{NaCl}$. In contrast, lower salt concentrations were sufficient to compete with the binding of $\mathrm{AAV}-\mathrm{V}_{\mathrm{EC}}$. In line, the addition of increasing concentrations of heparin to $\mathrm{AAV} 2$ and $\mathrm{AAV}-\mathrm{V}_{\mathrm{EC}}$ before cell transduction revealed that a lower concentration of heparin is

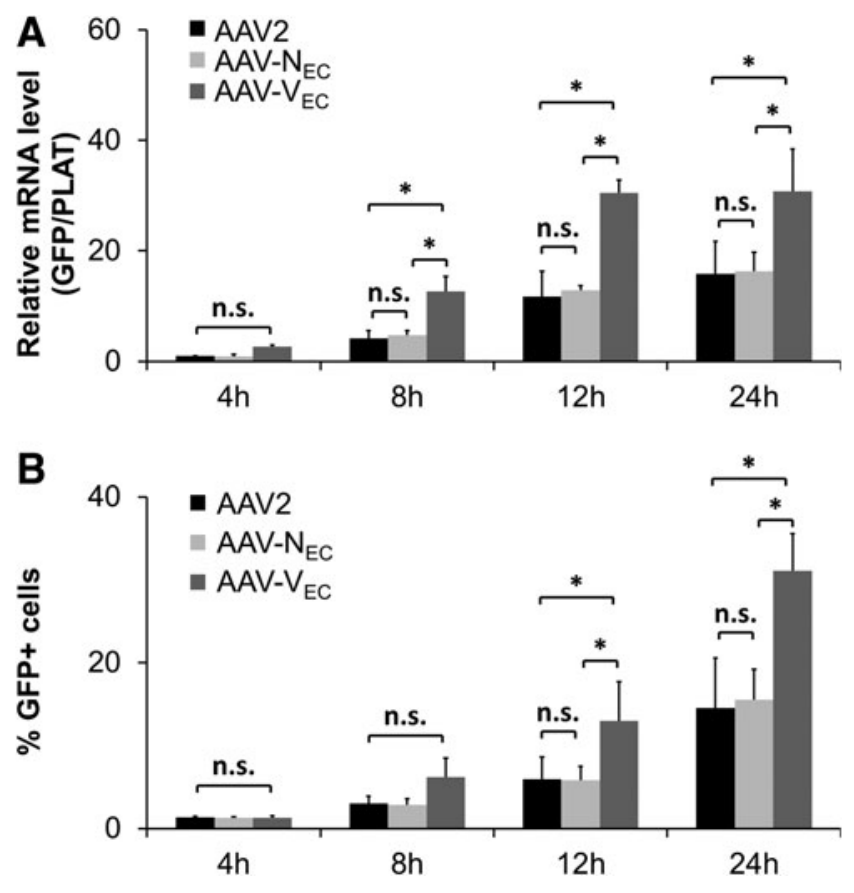

Figure 7. $A A V-V_{E C}$ does show a fast onset of transgene expression HUVEC were incubated with AAV2 (black), AAV- $\mathrm{N}_{\mathrm{EC}}$ (light gray), or AAV- $\mathrm{V}_{\mathrm{EC}}$ (dark gray) encoding for GFP in a single-stranded vector genome conformation at a $\mathrm{GOI}$ of 10,000. (A) Quantification of transgene-specific mRNA Total RNA was isolated at the indicated time points p.t., and GFP expression levels were determined by qPCR using GFP-specific primers. Values were normalized to GAPDH. (B) Quantification of transgene-expressing cells Cells were harvested at the indicated time points p.t., and percentage of transgene expressing cells was determined by flow cytometry. Shown are results of three independent experiments \pm SD. ${ }^{*} p<0.05$. 

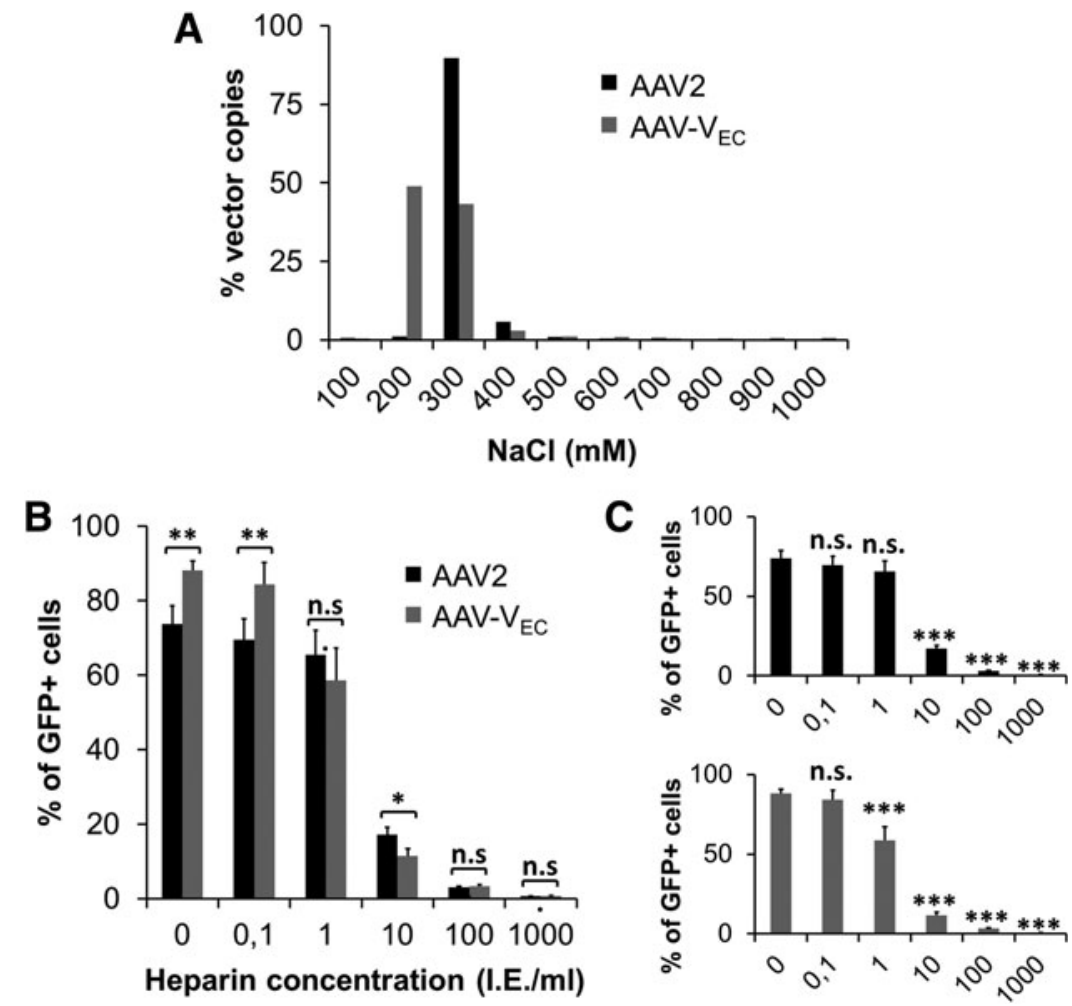

Figure 8. AAV-V $V_{E C}$ does bind heparin and thus HSPG. (A) AAV-V $V_{E C}$ shows lower affinity to heparin compared with AAV2. Heparin affinity chromatography was performed for AAV2 (black) and AAV- $\mathrm{V}_{\mathrm{EC}}$ (dark gray) vector stocks. Ten fractions were collected by using elution buffers with increasing ionic strength. For each fraction, five column volumes were collected. VCN were measured by qPCR using GFP-specific primers. Percentage of VCN was calculated as relative vector copies by setting total VCN to $100 \%$. Shown are results of a single experiment. (B, C) Cell transduction by AAV-V $\mathrm{EC}_{\mathrm{EC}}$ compared with AAV2 is competed by lower concentration of heparin. HUVEC were incubated with AAV2 (black) or AAV- $\mathrm{V}_{\mathrm{EC}}$ (dark gray) delivering an sc vector genome encoding for GFP controlled by a CMV promoter with a $\mathrm{GOI}$ of 10,000, in the presence of different concentrations of heparin. Percentage of GFP-expressing cells was determined $24 \mathrm{~h}$ p.t. by flow cytometry. The statistical analysis comparing the transduction efficiencies between AAV2 and AAV-V $V_{E C}$ is shown in (A). The statistical analysis comparing the different concentrations of heparin to control, for AAV2 (upper panel) and AAV- $V_{E C}$ (lower panel) is shown in (B). Shown are results of four independent experiments using two different batches for each vector \pm SD. ${ }^{*} p<0.05 ;{ }^{* *} p<0.01 ;{ }^{* * *} p<0.001$. VCN, vector copy numbers.

sufficient to interfere with $\mathrm{AAV}-\mathrm{V}_{\mathrm{EC}}$-mediated transduction of HUVEC (Fig. 8B, C).

AAVR is an essential receptor for the large majority of AAV serotypes to transduce human cells and mouse tissues. ${ }^{16,43}$ Recently, two groups mapped for AAV2 the AAVR binding site to the spike region adjacent to the threefold axis of symmetry. ${ }^{44,45}$ Since our capsid modification concerns the tip of the second highest spike, we were interested in determining whether AAV- $\mathrm{V}_{\mathrm{EC}}$ differs from AAV2 regarding the dependency on AAVR for cell transduction. In the absence of an EC-specific AAVR knockout cell line, we used HEK293-AAVR-KO and HeLa-AAVR-KO as model systems. These cells, and as control parental AAVR wild-type cells, were transduced with increasing doses of AAV- $\mathrm{V}_{\mathrm{EC}}$ and

SF5 AAV2, respectively (Supplementary Fig. S5). As expected, in the absence of AAVR, the transduction efficiency of AAV2 was reduced to background. AAV-V $V_{E C}$ also transduced both parental cell types, but neither in HEK293-AAVR-KO nor in HeLaAAVR-KO transgene expression above background was observed, revealing that at least for these otherwise highly permissive cell lines AAVR is an essential factor for AAV2 as well as for AAV- $\mathrm{V}_{\mathrm{EC}}$.

Overall, these results indicate that $A A V-V_{E C}$ binds heparin/HSPG, although with a lower affinity compared with AAV2. Based on the strong selection for $R$ in position 7 of inserted peptides, we might argue that binding to HSPG or other negatively charged cell surface molecules is an advantage for transducing EC. However, binding to HSPG is also correlated with a board tropism (Uhrig et $a l^{42}$ and Supplementary Fig. S5) and accumulation in liver tissue when vectors are applied intravenously. ${ }^{41}$ Thus, AAV-V $\mathrm{E}_{\mathrm{EC}}$ might be best suited for local in vivo application or ex vivo transduction of EPC that are subsequently infused in case an EC-selective transduction is desired.

\section{$A A V-V_{E C}$ transduces quiescent EC with higher efficiency than AAV2 or AAV- $\mathrm{N}_{\mathrm{EC}}$}

Proliferating EC as used in this study are located at the tip of sprouting vessels under physiological 
conditions (i.e., wound healing) and during tumorinduced angiogenesis, whereas the majority of EC lining the inner part of vessels are nonproliferating. To mimic these conditions, quiescence was induced by depleting supplements and FBS from culture medium as indicated by reduced incorpora-

F9 tion of EdU (Fig. 9A vs. B). Side-by-side comparison of AAV2, AAV- $\mathrm{V}_{\mathrm{EC}}$, and AAV- $\mathrm{N}_{\mathrm{EC}}$ demonstrated that $\mathrm{AAV}-\mathrm{V}_{\mathrm{EC}}$ was the most efficient vector with exception of transduction at GOI 2000 (Fig. 9C). For AAV-N $\mathrm{EC}_{\mathrm{EC}}$, an advantage compared with AAV2 was again only observed at the highest vector dose. However, transduction efficiency for AAV2, AAV$\mathrm{V}_{\mathrm{EC}}$, and AAV- $\mathrm{N}_{\mathrm{EC}}$ on quiescent cells was lower compared with proliferating cells.

\section{iPSC-derived EPC are highly susceptible toward AAV- $\mathrm{V}_{\mathrm{EC}}$ transduction}

Ex vivo genetic modification of EPC followed by re-infusion might represent a promising alternative to direct in vivo modification, because EPC home to sites of tissue damage or tumor growth. ${ }^{46-}$ 48 Consequently, we tested iPSC-derived EPC (iEC), which are generated in a patient-specific manner, but they also hold promise as a future source for off-the-shelf EPC, regarding their transducibility with AAV vectors. Specifically, a human fibroblast-derived iPSC clone was differentiated into iEC by using a forward reprogramming protocol. ${ }^{24}$ At days 8 and 14 of differentiation,
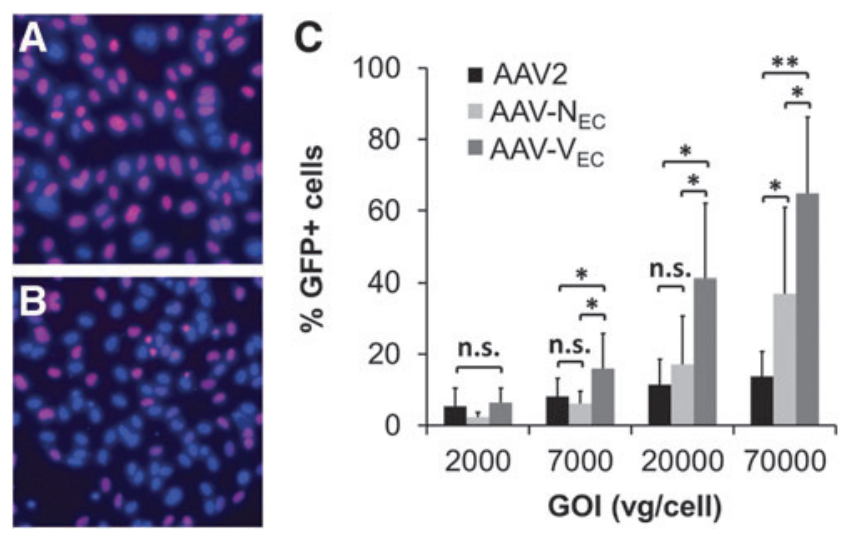

Figure 9. Quiescent HUVEC are permissive, in particular, for AAV-V $V_{E C}$. (A, B) EdU staining of HUVEC. (A) Proliferating HUVEC and (B) HUVEC after induction of quiescence through depletion of supplements and fetal bovine serum were fixed. Proliferation is marked by EdU incorporation (red stained

AU16 cells). Cell nuclei were stained by DAPI (blue stained cells). (C) Trans-

$4 \mathrm{C}$ duction of quiescent cells. Low-passage HUVEC were incubated with a serial dilution of AAV2 (black), AAV- $\mathrm{N}_{\mathrm{EC}}$ (light gray), or AAV- $\mathrm{V}_{\mathrm{EC}}$ (dark gray). Vectors encode for GFP controlled by CMV promoter in a single-stranded vector genome conformation. GOI is indicated. Percentage of transgene (GFP)-expressing cells was determined by flow cytometry $24 \mathrm{~h}$ p.t. Experiments were performed three times independently. Error bars indicate SD. ${ }^{* *} p<0.001 ;{ }^{*} p<0.05$. EdU, 5 -ethynyl-2'-deoxyuridine.
$\mathrm{iEC}$ were incubated with $\mathrm{AAV}-\mathrm{V}_{\mathrm{EC}}$ and $\mathrm{AAV} 2$ vectors at a GOI of 200 , followed by transduction efficiency analyses $48 \mathrm{~h}$ later (Fig. 10).

\section{$<\mathrm{F} 10$}

At both stages of differentiation, iEC were susceptible toward AAV2 and AAV- $\mathrm{V}_{\mathrm{EC}}$. AAV-V $\mathrm{V}_{\mathrm{EC}}$; however, transducing iEC with, for example, $76.1 \pm 1.9 \%$ of transduced iEC (d14) was clearly superior versus $20.8 \pm 1.3 \%$ for AAV2. This high efficiency of transduction prompted us to assay even lower GOI (Supplementary Fig. S6). Sur- 4 SF6 prisingly, with a GOI as low as $50,>40 \%$ of AAV$\mathrm{V}_{\mathrm{EC}}$ treated cells showed reporter gene expression clearly above background. Transduction efficiency of $\mathrm{AAV}-\mathrm{V}_{\mathrm{EC}}$ could be increased to nearly $100 \%$ with a GOI of only 1000 (Supplementary Fig. S6).

\section{DISCUSSION}

EC form the inner layer of vessels controlling vasomotor tone, transport, and permeability. ${ }^{49,50}$ In addition, EC contribute to the innate immune response, possess key functions in vascular homeostasis, and secrete proteinous factors into the blood stream. ${ }^{49-51}$ Consequently, disruption of the EC layer or of vascular homeostasis triggers disease processes, making EC attractive targets for therapeutic interventions. ${ }^{50}$ The latter include genetic modification in gene therapy or regenerative medicine settings employing the homing capability of EC to repair damaged tissue or to target growing (tumor) vessels. ${ }^{15}$ EC are, however, only weakly susceptible to the most common viral vectors. ${ }^{27}$ Here, we focused on the AAV vector system and developed an AAV variant, $\mathrm{AAV}-\mathrm{V}_{\mathrm{EC}}$, for improving transduction of proliferating, quiescent,
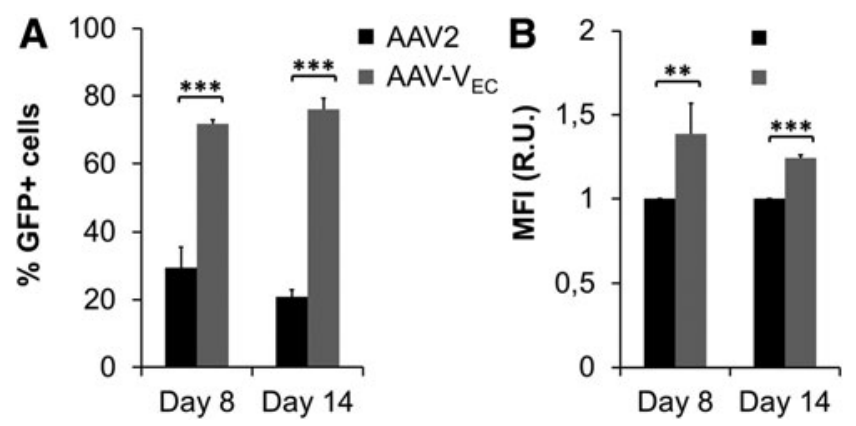

Figure 10. Transduction of $\mathrm{iEC}$ at days 8 and 14 of differentiation. The human fibroblast-derived iPSC clone $\mathrm{H}_{2} \mathrm{E} \mathrm{C}^{24}$ was differentiated into iEC. At days 8 and 14 of differentiation, iEC were incubated with AAV2 (black) or AAV- $V_{E C}$ (dark gray). Vectors delivered a self-complementary vector genome encoding for GFP controlled by a CMV promoter. Transduction efficiency and transgene expression level was determined by flow cytometry $48 \mathrm{~h}$ p.t. (A) Percentage of GFP-expressing cells and (B) MFI. In $B$, values obtained for AAV2 are set to one. Shown are results of three independent experiments \pm SD. ${ }^{* *} p<0.01 ;{ }^{* * *} p<0.001$. iEC, iPSC-derived endothelial progenitor cells; iPSC, induced pluripotent stem cell; MFI, mean fluorescence intensity. 
and iPSC-derived EC. As this variant clearly outperformed the parental serotype, we used this variant (and $\mathrm{AAV}-\mathrm{N}_{\mathrm{EC}}$ ) to identify barriers that limit transduction of EC.

AAV vector transduction is a multistep process that is initiated by binding to cell surface glycans such as sialic acids in case of AAV1, $-4,-5$, and -6 , or HSPG in case of AAV2, the serotype we used as the basis for our study. ${ }^{52}$ This attachment primes vector internalization mediated by co-receptors such as $\alpha \mathrm{v} \beta 5$ or $\alpha 5 \beta 1$ integrins. ${ }^{30,31}$ Antibody staining confirmed the presence of both integrins, whereas HSPG was barely detectable (Fig. 1B). This condition did not impair vector uptake since AAV2 vector particles were detected intracellularly but seemed to limit efficacy of AAV2 internalization (Fig. 6, Supplementary Fig. S3), because with AAV-V $V_{E C}$, a vector that uses its inserted peptide for cell entry, significantly higher VCN were detected (Fig. 6, Supplementary Fig. S3). AAV2, AAV-V $\mathrm{EC}_{\text {, }}$ and $\mathrm{AAV}-\mathrm{N}_{\mathrm{EC}}$ were trafficked to the nucleus, although significantly more capsid-engineered AAV than AAV2 particles reached the nucleus (Fig. 6). Interestingly, nuclear accumulation did not correlate with transduction efficiency. Specifically, AAV-V $\mathrm{V}_{\mathrm{EC}}$ and $\mathrm{AAV}-\mathrm{N}_{\mathrm{EC}}$ differed remarkably regarding the onset of transgene expression as well as the level of transgene expression, although both vectors exhibited a comparable extent of nuclear trafficking (Figs. 3, 6, and 7). Transgene expression requires availability of vector genomes for transcription. As vectors delivered identical vector genomes, AAV$\mathrm{V}_{\mathrm{EC}}$ seems to be more efficient in releasing vector genomes. This feature is may be due to the specific peptide insert, VSSSTPR, or the receptor and respective downstream signaling pathways activated on binding of this engineered capsid to the EC surface or a combination of both mechanisms. Once released from the capsid, single-stranded AAV vector genomes need to be converted into a doublestranded DNA for initiation of transcription. Since $\mathrm{AAV}-\mathrm{V}_{\mathrm{EC}}$ applied in the expression kinetics experiment (Fig. 7) delivered a single-stranded AAV vector genome from which transcribed mRNA is detectable by $\mathrm{qPCR}$ already at early time points p.t., EC-at least HUVEC-possess all necessary components to assist AAV in second-stranded synthesis. Nevertheless, using a self-complementary instead of the natural single-stranded vector genome conformation is advantageous, particularly when only a few vector genomes are present, that is, in conditions of low GOI (Fig. 4).

Thus, regarding the main steps in AAV infection-cell surface attachment, internalization, trafficking toward the nuclear area, release from the endosomal compartment, nuclear delivery, uncoating, and second-strand synthesis-vector uptake and uncoating appear as the most critical steps that impact EC transduction, whereas use of a self-complementary vector genome conformation can further improve transduction efficiency.

$A A V-V_{E C}$, selected by an AAV peptide display screen on HUVEC, differs from AAV2 in the 7mer peptide VSSSTPR inserted at position 587 of each of the 60 subunits of the AAV capsid. Peptides inserted at that position were demonstrated to mediate receptor binding and determine vector tropism. ${ }^{5}$ The receptor for $\mathrm{AAV}-\mathrm{V}_{\mathrm{EC}}$ is currently unknown, a general caveat of directed evolution approaches in which variants are selected for tropism for a predefined target cell population without the option to predefine the target receptor. The latter-if required-needs to be determined afterward, for example, by comparative gene analyses. ${ }^{8}$ Despite this drawback, high-throughput selection screens of AAV capsid libraries have-since their first application more than 15 years ago ${ }^{11,12}$-demonstrated their potency to tailor the AAV-target cell interaction without the need to know barriers that limit respective target cell transduction. ${ }^{1,13,53}$ As mentioned earlier, the adaptation in AAV-EC interaction regarding $\mathrm{AAV}-\mathrm{V}_{\mathrm{EC}}$ concerned vector uptake (compared with AAV2) and uncoating (compared with $A A V-N_{E C}$ ). Comparison of AAV-V $V_{E C}$ with AAVSIG, a first-generation capsid-engineered vector that displayed a peptide identified by phage display selection on HUVEC, revealed a 13-fold higher transduction efficiency for AAV-V $\mathrm{V}_{\mathrm{EC}}$ (Fig. 5A). This is likely due to the fact that selection of the SIGYPLP peptide was based on overcoming only one barrier, that is, the uptake process, since the SIGYPLP peptide was selected for improved binding to EC without tackling further downstream barriers. Our capsid variant selected here also demonstrated superior transduction efficiency compared with AAV-NADARVS (Fig. 5B). Since we did not perform a side-by-side comparison of $\mathrm{AAV}-\mathrm{V}_{\mathrm{EC}}$ and AAV-NADARVS for the various steps of EC transduction, we can only speculate on the reason(s) for this observation. It is possible that the NADARVS peptide is underestimated in our case, as we cloned this peptide into position 587, whereas Muller et al. selected the peptide from an AAV peptide display library displaying peptides at position 588, the neighboring position. ${ }^{12}$ This peptide, however, mediated EC targeting with a comparable efficiency when displayed at an AAV9 capsid, which argues against a positional effect. ${ }^{28}$ Alternatively, improved efficiency by AAV-NADARVS may be restricted to human coronary EC, the EC subtype this 
variant has been selected on, and/or requires cofactors since selection of AAV-NADARVS was performed in the presence of adenovirus, whereas we decided against adenovirus superinfection.

We did not perform extensive screenings for EC subtypes that were susceptible to $\mathrm{AAV}-\mathrm{V}_{\mathrm{EC}}$, but we demonstrated $\mathrm{AAV}-\mathrm{V}_{\mathrm{EC}}$ 's ability to transduce $\mathrm{HU}$ VEC independent of their proliferation state as well as iPSC-derived EPC (Figs. 3, 9, and 10). The latter are currently entering the arena of drug screening as they allow patient-specific disease modeling. ${ }^{50}$ In addition, EPC home to damaged vasculature, making them promising targets for genetic modification to develop novel therapeutic strategies in the context of regenerative medicine, gene therapy, organ transplantation, and cardiovascular disease $^{54}$ as well as antitumor therapy. ${ }^{55}$ Given the recent advancement in iPSC technology, in particular, with regard to "off the shelf cell products," iPSC-derived EPC will likely replace conventional EPC in the clinical setting. With its impressive efficacy in transducing iPSC-derived $\mathrm{EPC}, \mathrm{AAV}-\mathrm{V}_{\mathrm{EC}}$ may represent a highly potent delivery system also in this setting.

In summary, we here report on the development of $A A V-V_{E C}$, a capsid-engineered $A A V$ vector, that is internalized and consequently transported to the nucleus with significantly higher efficacy than the parental serotype. AAV- $\mathrm{V}_{\mathrm{EC}}$ showed efficient transduction of conventional EC at different states of differentiation and also demonstrated an impressive efficacy in iPSC-derived EPC. Of note, efficient and fast onset in transgene expression was observed even when delivering the vector genome in its natural single-stranded DNA conformation. This option is particularly promising for clinical application, as the full coding capacity of the AAV vector system is thereby available for delivery of transgene expression cassettes of $\sim 5$ instead of
$2.5 \mathrm{~kb}$, the upper limit of AAV vectors with a selfcomplementary vector genome design.

\section{ACKNOWLEDGMENTS}

The authors thank Jude Samulski (University of North Carolina at Chapel Hill, NC) for providing plasmid pXX6. They also thank Hanna Janicki (University of Cologne, Germany), Claudia Vollbrecht (University of Cologne, Germany), Nicki Lenort (Hannover Medical School), and Elke Barczak (Hannover Medical School) for their excellent technical assistance.

\section{AUTHOR DISCLOSURE}

No competing financial interests exist.

\section{FUNDING INFORMATION}

This work was supported by grants from Uni- 4 AU14 versity Hospital Cologne (Köln Fortune) to H.B. and M.O., the Center for Molecular Medicine Cologne (CMMC) to H.B., BMBF and MWK Lower Saxony-funded Professorinnenprogramm Niedersachsen to H.B., the DFG-funded KFO286 to H.B., the DFG-funded cluster of excellence REBIRTH to A.S. and H.B., and the DFG-funded SFB738 to A.S. L.Z. held a scholarship from the German Academic Exchange Service (DAAD).

\section{SUPPLEMENTARY MATERIAL}

Supplementary Figure S1

Supplementary Figure S2

Supplementary Figure S3

Supplementary Figure S4

Supplementary Figure S5

Supplementary Figure S6

Supplementary Table S1

Supplementary Table S2

\section{REFERENCES}

1. Grimm D, Buning H. Small but increasingly mighty: latest advances in AAV vector research, design, and evolution. Hum Gene Ther 2017;28:1075-1086.

2. Domenger C, Grimm D. Next-generation AAV vectors - don't judge a virus (only) by its cover. Hum Mol Genet 2019 [Epub ahead of print]; DOI: 10.1093/hmg/ddz148.

3. Keeler AM, Flotte TR. Recombinant adenoassociated virus gene therapy in light of luxturna (and zolgensma and glybera): where are we, and how did we get here? Annu Rev Virol 2019 [Epub ahead of print]; DOI: 10.1146/annurev-virology092818-015530.
4. Colella P, Ronzitti G, Mingozzi F. Emerging issues in AAV-mediated in vivo gene therapy. Mol Ther Methods Clin Dev 2018:8:87-104.

5. Buning $H$, Huber $A$, Zhang $L$, et al. Engineering the AAV capsid to optimize vector-host-interactions. Curr Opin Pharmacol 2015;24:94-104.

6. Nathwani AC, Reiss UM, Tuddenham EG, et al. Long-term safety and efficacy of factor IX gene therapy in hemophilia B. N Engl J Med 2014;371: 1994-2004.

7. Mendell JR, Al-Zaidy S, Shell R, et al. Single-dose gene-replacement therapy for spinal muscular atrophy. N Engl J Med 2017;377:1713-1722.
8. Sallach J, Di Pasquale G, Larcher F, et al Tropism-modified AAV vectors overcome barriers to successful cutaneous therapy. Mol Ther 2014; 22:929-939.

9. Pandya J, Ortiz L, Ling C, et al. Rationally designed capsid and transgene cassette of AAV6 vectors for dendritic cell-based cancer immunotherapy. Immunol Cell Biol 2014;92:116-123.

10. Buchholz CJ, Friedel T, Buning H. Surface-engineered viral vectors for selective and cell type-specific gene delivery. Trends Biotechnol 2015;33:777-790.

11. Perabo L, Buning $H$, Kofler DM, et al. In vitro selection of viral vectors with modified tropism: 
the adeno-associated virus display. Mol Ther 2003:8:151-157

12. Muller OJ, Kaul F, Weitzman MD, et al. Random peptide libraries displayed on adeno-associated virus to select for targeted gene therapy vectors. Nat Biotechnol 2003;21:1040-1046.

13. Weinmann J, Grimm D. Next-generation AAV vectors for clinical use: an ever-accelerating race. Virus Genes 2017:53:707-713.

14. Naumer M, Popa-Wagner R, Kleinschmidt JA. Impact of capsid modifications by selected peptide ligands on recombinant adeno-associated virus serotype 2-mediated gene transduction. J Gen Virol 2012;93:2131-2141.

15. Hristov M, Weber C. Endothelial progenitor cells in vascular repair and remodeling. Pharmacol Res 2008:58:148-151.

16. Pillay S, Meyer NL, Puschnik AS, et al. An essential receptor for adeno-associated virus infection. Nature 2016:530:108-112.

17. Kofler DM, Buning H, Mayr C, et al. Engagement of the B-cell antigen receptor (BCR) allows efficient transduction of ZAP-70-positive primary BCLL cells by recombinant adeno-associated virus (rAAV) vectors. Gene Ther 2004;11:1416-1424.

18. Nicklin SA, Buening $\mathrm{H}$, Dishart $\mathrm{KL}$, et al. Efficient and selective AAV2-mediated gene transfer directed to human vascular endothelial cells. Mol Ther 2001;4:174-181.

19. Girod A, Ried M, Wobus C, et al. Genetic capsid modifications allow efficient re-targeting of adenoassociated virus type 2. Nat Med 1999;5:1052-1056.

20. Hacker UT, Wingenfeld L, Kofler DM, et al. Adenoassociated virus serotypes 1 to 5 mediated tumor cell directed gene transfer and improvement of transduction efficiency. J Gene Med 2005;7:1429_ 1438

21. Hacker UT, Gerner FM, Buning H, et al. Standard heparin, low molecular weight heparin, low molecular weight heparinoid, and recombinant hirudin differ in their ability to inhibit transduction by recombinant adeno-associated virus type 2 vectors. Gene Ther 2001;8:966-968.

22. Xiao X, Li J, Samulski RJ. Production of high-titer recombinant adeno-associated virus vectors in the absence of helper adenovirus. J Virol 1998;72: 2224-2232.

23. Hosel M, Lucifora J, Michler T, et al. Hepatitis B virus infection enhances susceptibility toward adeno-associated viral vector transduction in vitro and in vivo. Hepatology 2014;59:2110-2120.

24. Hoffmann D, Schott JW, Geis FK, et al. Detailed comparison of retroviral vectors and promoter configurations for stable and high transgene expression in human induced pluripotent stem cells. Gene Ther 2017:24:298-307.

25. Orlova WV, Drabsch Y, Freund C, et al. Functionality of endothelial cells and pericytes from human pluripotent stem cells demonstrated in cultured vascular plexus and zebrafish xenografts. Arter Thromb Vasc Biol 2014:34:177-186.
26. Rossi A, Dupaty L, Aillot L, et al. Vector uncoating limits adeno-associated viral vector-mediated transduction of human dendritic cells and vector immunogenicity. Sci Rep 2019;9:3631.

27. Sen S, Conroy S, Hynes SO, et al. Gene delivery to the vasculature mediated by low-titre adenoassociated virus serotypes 1 and 5. J Gene Med 2008:10:143-151.

28. Varadi K, Michelfelder S, Korff T, et al. Nove random peptide libraries displayed on AAV serotype 9 for selection of endothelial cell-directed gene transfer vectors. Gene Ther 2012;19:800-809.

29. Summerford C, Samulski RJ. Membraneassociated heparan sulfate proteoglycan is a receptor for adeno-associated virus type 2 virions. J Virol 1998;72:1438-1445.

30. Asokan A Hamra JB, Govindasamy L, et al. Adeno-associated virus type 2 contains an integrin alpha5beta1 binding domain essential for viral cell entry. J Virol 2006;80:8961-8969.

31. Summerford C, Bartlett JS, Samulski RJ. AlphaVbeta5 integrin a co-receptor for adeno-associated virus type 2 infection. Nat Med 1999:5:78-82.

32. Levy HC, Bowman VD, Govindasamy L, et al. Heparin binding induces conformational changes in Adeno-associated virus serotype 2. J Struct Biol 2009;165:146-156.

33. McCarty DM. Self-complementary AAV vectors; advances and applications. Mol Ther 2008:16: 1648-1656.

34. White SJ, Nicklin SA, Buning $\mathrm{H}$, et al. Targeted gene delivery to vascular tissue in vivo by tropismmodified adeno-associated virus vectors. Circulation 2004;109:513-519.

35. Work LM, Buning $H$, Hunt $E$, et al. Vascular bedtargeted in vivo gene delivery using tropismmodified adeno-associated viruses. Mol Ther 2006;13:683-693

36. White $K$, Buning $H$, Kritz $A$, et al. Engineering adeno-associated virus 2 vectors for targeted gene delivery to atherosclerotic lesions. Gene Ther 2008;15:443-451.

37. Waterkamp DA, Muller OJ, Ying Y, et al. Isolation of targeted AAV2 vectors from novel virus display libraries. J Gene Med 2006;8:1307-1319.

38. Naumer M, Ying Y, Michelfelder S, et al. Development and validation of novel AAV2 random libraries displaying peptides of diverse lengths and at diverse capsid positions. Hum Gene Ther 2012;23:492-507.

39. Korbelin J, Sieber T, Michelfelder S, et al. Pulmonary targeting of adeno-associated viral vectors by next-generation sequencing-guided screening of random capsid displayed peptide libraries. Mol Ther 2016;24:1050-1061.

40. Buning $H$, Srivastava A. Capsid modifications for targeting and improving the efficacy of AAV vectors. Mol Ther Methods Clin Dev 2019;12:248-265.

41. Perabo L, Goldnau D, White K, et al. Heparan sulfate proteoglycan binding properties of adeno-associated virus retargeting mutants and consequences for their in vivo tropism. J Virol 2006:80:7265-7269.
42. Uhrig S, Coutelle 0, Wiehe T, et al. Successful target cell transduction of capsid-engineered rAAV vectors requires clathrin-dependent endocytosis. Gene Ther 2012;19:210-218.

43. Dudek AM, Pillay S, Puschnik AS, et al. An alternate route for adeno-associated virus (AAV) entry independent of AAV receptor. J Virol 2018;92:e02213-17.

44. Meyer NL, Hu G, Davulcu O, et al. Structure of the gene therapy vector, adeno-associated virus with its cell receptor, AAVR. Elife 2019;8:e44707.

45. Zhang R, Cao L, Cui M, et al. Adeno-associated virus 2 bound to its cellular receptor AAVR. Nat Microbiol 2019;4:675-682

46. Laurenzana A, Biagioni A, D'Alessio S, et al. Melanoma cell therapy: endothelial progenitor cells as shuttle of the MMP12 uPAR-degrading enzyme. Oncotarget 2014;5:3711-3727.

47. Purwanti YI, Chen C, Lam DH, et al. Antitumor effects of CD40 ligand-expressing endothelial progenitor cells derived from human induced pluripotent stem cells in a metastatic breast cancer model. Stem Cells Transl Med 2014;3:923-935.

48. Zhang JX, Kang CS, Shi L, et al. Use of thymidine kinase gene-modified endothelial progenitor cells as a vector targeting angiogenesis in glioma gene therapy. Oncology 2010;78:94-102.

49. Wiktorowska-Owczarek A, Namiecinska M, Balcerczyk $A$, et al. Human micro- and macrovesselderived endothelial cells: a comparative study on the effects of adrenaline and a selective adenosine A2-type receptor agonist under normoxic and hypoxic conditions. Pharmacol Rep 2007;59:800-806.

50. Cochrane A, Albers HJ, Passier R, et al. Advanced in vitro models of vascular biology: human induced pluripotent stem cells and organ-on-chip technology. Adv Drug Deliv Rev 2019;140:68-77.

51. Viemann D, Goebeler M, Schmid S, et al. TNF induces distinct gene expression programs in microvascular and macrovascular human endothelial cells. J Leukoc Biol 2006;80:174-185.

52. Mietzsch M, Broecker F, Reinhardt A, et al. Differential adeno-associated virus serotype-specific interaction patterns with synthetic heparins and other glycans. J Virol 2014;88:2991-3003.

53. Kotterman MA, Schaffer DV. Engineering adenoassociated viruses for clinical gene therapy. Nat Rev Genet 2014;15:445-451.

54. Devanesan AJ, Laughlan KA, Girn HR, et al. Endothelial progenitor cells as a therapeutic option in peripheral arterial disease. Eur J Vasc Endovasc Surg 2009;38:475-481.

55. Werling NJ, Thorpe R, Zhao Y. A systematic approach to the establishment and characterization of endothelial progenitor cells for gene therapy. Hum Gene Ther Methods 2013;24:171-184.

Received for publication Febraury 5, 2019; accepted after revision July 27, 2019

Published online: August 9, 2019 


\section{Supplementary Data}

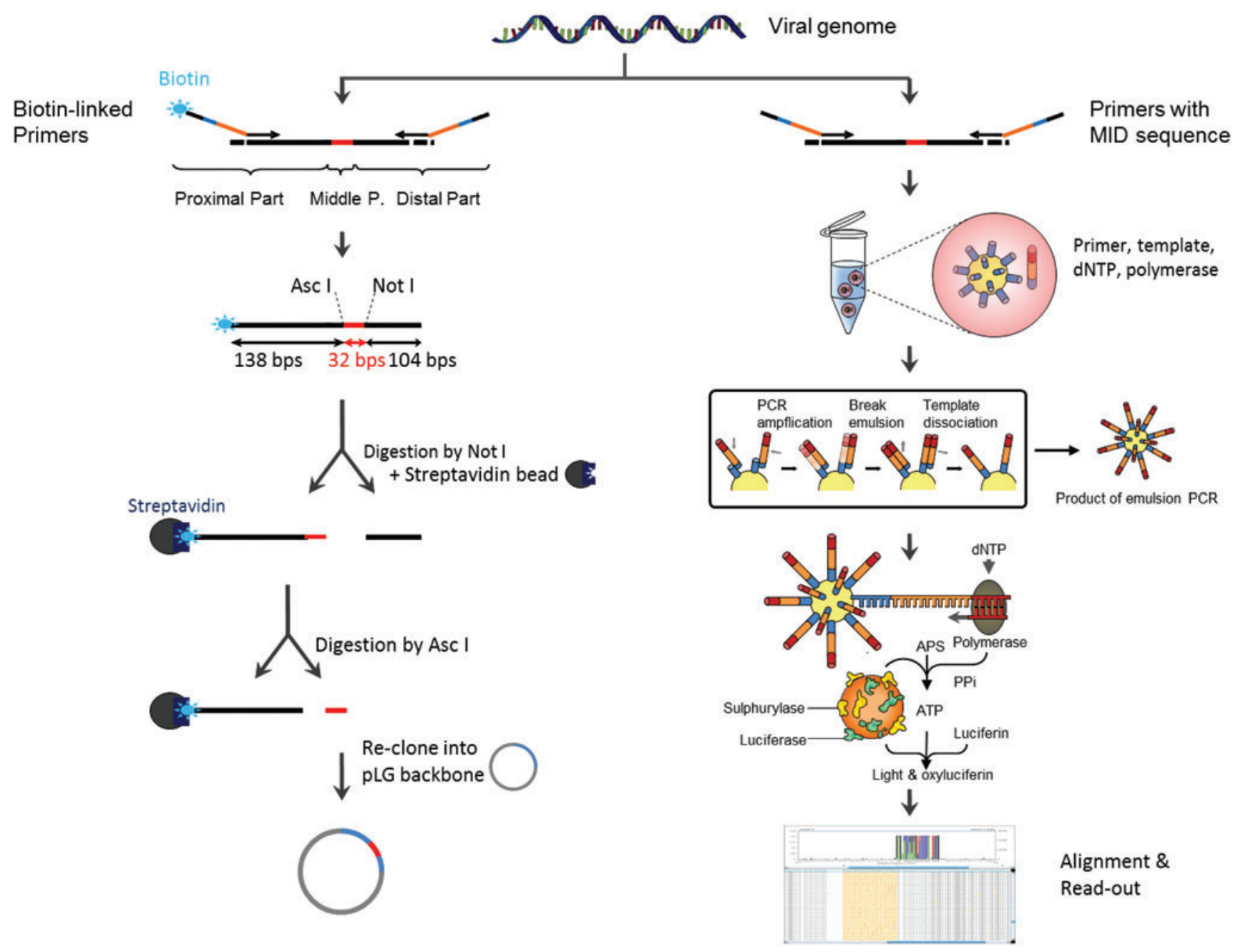

Supplementary Figure S1. NGS-based monitoring and cloning procedure. Total DNA isolated from library-treated cells. NGS primers are designed to recognize unique cap sequence flanking the random sequence coding for the 7 mer peptide and restriction enzyme recognition sites. (left panel) Molecular cloning of sub-library. The forward NGS primer has been modified with biotin, whereas the backward NGS primer remained unmodified. After the PCR amplification step, DNA amplicons are carrying a biotin residue at the $5^{\prime}$-end and contain the random peptide encoding sequences flanked by Ascl and Notl restriction enzyme recognition sites. These two restriction sites are the same that were used for insertion of the random oligonucleotides for the initial library cloning. DNA amplicons are treated with Notl and are purified by streptavidin microbead-mediated pull-down followed by Ascl-mediated release of the amplicons from the beads. The latter were then used for ligation and generation of the sub-library. (right panel) Monitoring of AAV library selection process. The same NGS primers (left panel, but now both unmodified) were used to monitor the selection process as such and the enrichment of specific clones to identify candidates for further characterization. AAV, adeno-associated viral; NGS, next generation sequencing; PCR, polymerase chain reaction. 


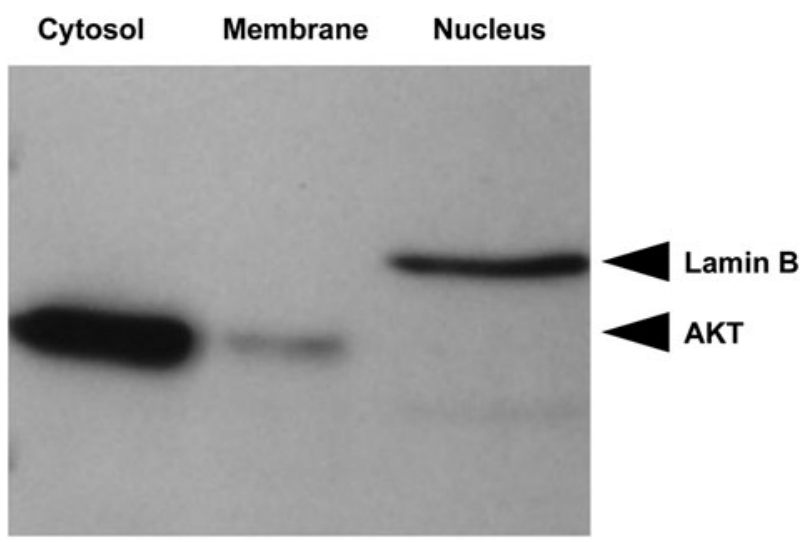

Supplementary Figure S2. Subcellular compartmentalization. HUVEC were subjected to cell compartmentalization according to the manufacturer's protocol. Proteins extracted from the cytosolic membrane and nuclear fractions were analyzed by western blotting using antibodies against AKT and Lamin B. HUVEC, human umbilical vein endothelial cells.
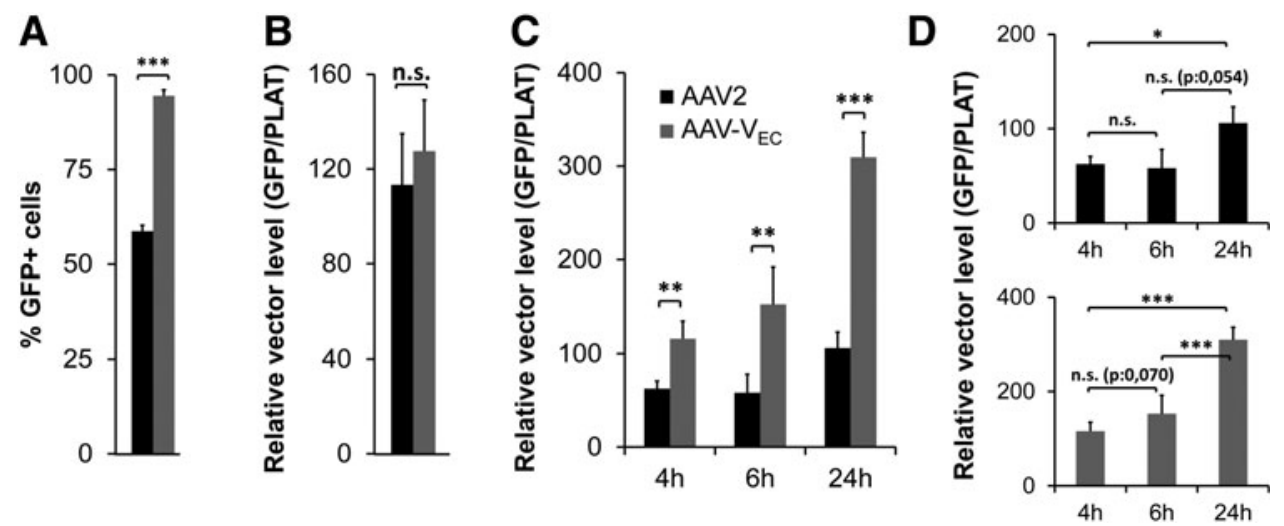

Supplementary Figure S3. AAV- $V_{E C}$ is internalized with higher efficiency, despite a similar binding ability compared with AAV2. HUVEC were incubated with AAV2 (black) or AAV- $\mathrm{V}_{\mathrm{EC}}$ (dark gray) delivering an sc vector genome encoding for GFP controlled by CMV promoter at a GOI of 5,000. Transduction was synchronized on ice for $1 \mathrm{~h}$. (A) Transduction efficiencies. Percentages of GFP-expressing cells were determined $24 \mathrm{~h}$ p.t. by flow cytometry. (B) Binding efficiencies. Before shifting to $37^{\circ} \mathrm{C}$, unbound particles were removed and cells were harvested. Total DNA was isolated, and VCN was quantified by qPCR using GFP primers and normalized against PLAT, which served as housekeeping gene. (C, D) Entry efficiencies. At the indicated time points, membrane-bound and unbound particles were removed, total DNA was isolated, and VCN was quantified by qPCR using GFP primers and normalized against PLAT. Statistical analysis comparing vectors is shown in (C), the one comparing the different time points for AAV2 (upper paneh) and AAV- $\mathrm{V}_{\mathrm{EC}}($ lower pane), is shown in (D). Shown are results of four independent experiments using two different batches for each vector \pm standard deviation. ${ }^{*} p<0.05$; ${ }^{* *} p<0.01$; ${ }^{* * *} p<0.001$. AAV2, AAV serotype 2; CMV, cytomegalovirus; GFP, green fluorescent protein; n.s., non-significant; PLAT, plasminogen activator; p.t., post transduction; qPCR, quantitative PCR; VCN, vector copy numbers. 


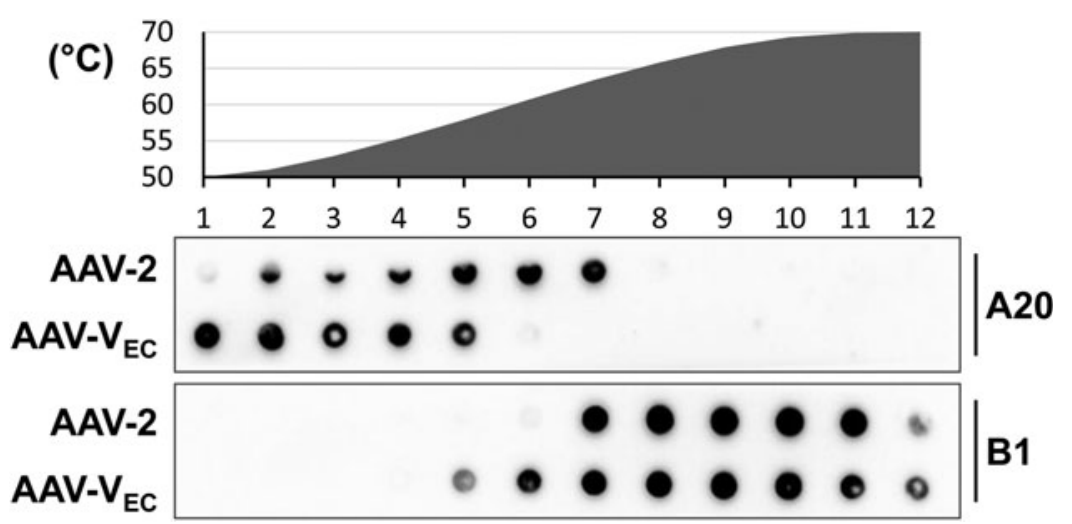

Supplementary Figure S4. AAV- $V_{E C}$ capsids show lower thermal stability compared with AAV2. AAV2 and AAV-VEC were submitted to a temperature gradient followed by a native dot blot analysis using A20 and B1 antibodies, allowing to discriminate intact and disassembled AAV capsids, respectively. Shown are results of a single experiment.

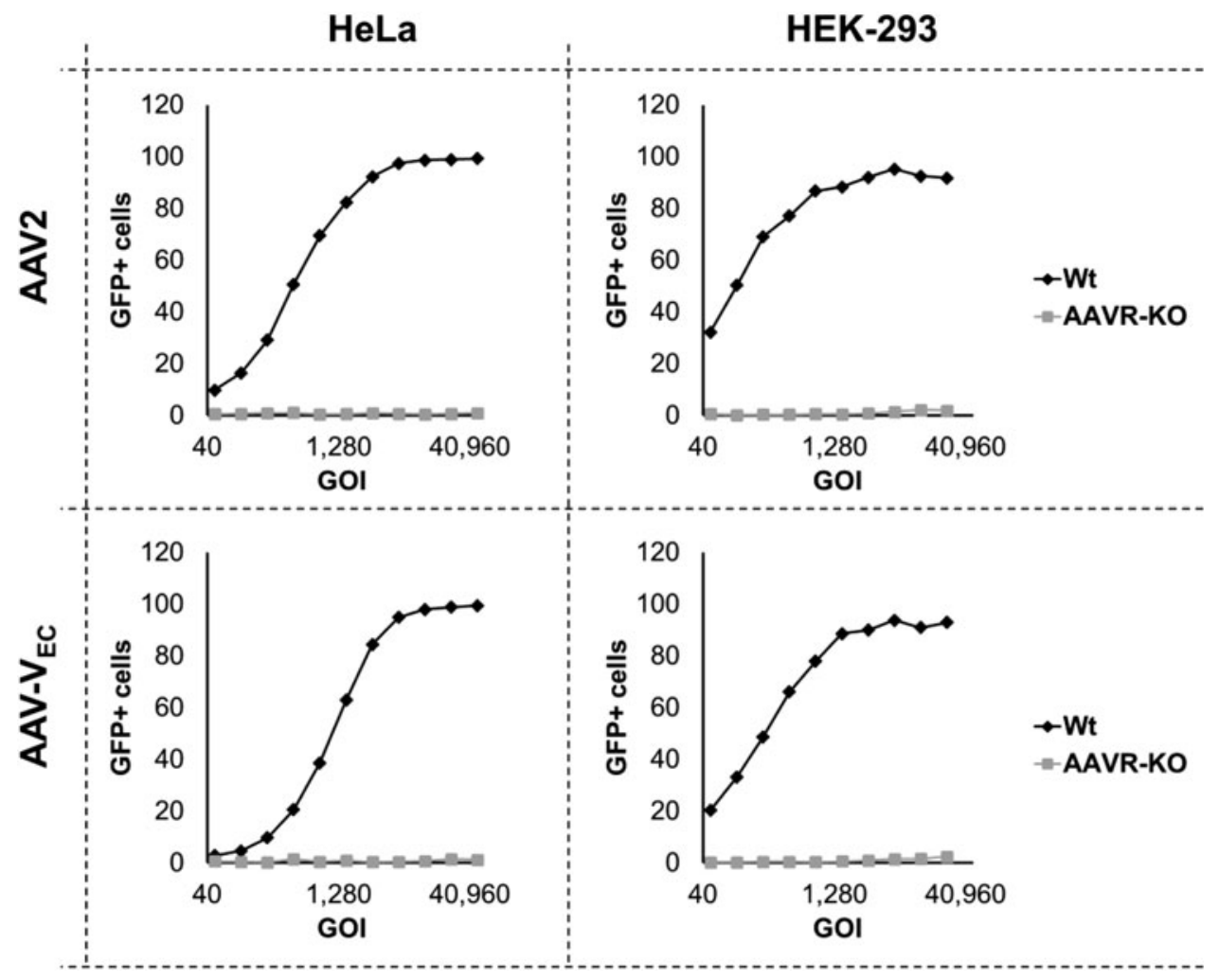

Supplementary Figure S5. AAV- $\mathrm{V}_{\mathrm{EC}}$ requires AAVR for efficient transduction of HeLa and HEK-293 cells. AAV2 (upper panels) or AAV-V $\mathrm{V}_{\mathrm{EC}}$ (lower pane/s) delivering an sc vector genome encoding for GFP controlled by CMV promoter were applied to HeLa (left pane/s) or HEK-293 (right pane/s) cell lines that express (wt) or not AAVR (AAVR-KO). Percentage of GFP-expressing cells was determined $24 \mathrm{~h}$ p.t. by flow cytometry. Shown are results of two independent experiments (using two batches of both vectors; mean value). AAVR-KO, AAV receptor knock-out. 


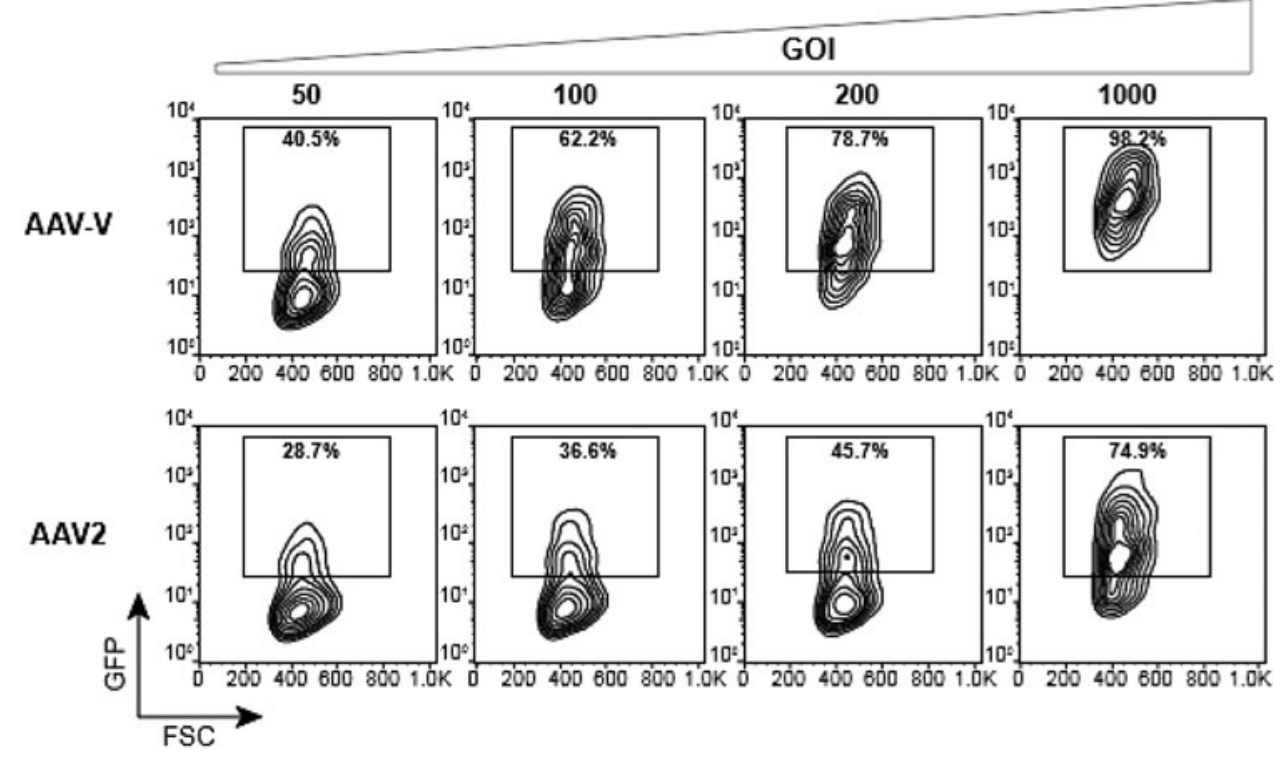

Supplementary Figure S6. Transduction of $\mathrm{EEC}$ with increasing vector GOI. iEC at day 8 of differentiation were incubated with indicated GOI of AAV-VEC and AAV2, respectively. AAV vector encoded for GFP controlled by CMV promoter in a self-complementary vector genome conformation. GOI, genome-per-cell ratio; iEC, induced pluripotent stem cell-derived endothelial progenitor cells.

Supplementary Table S1. NGS primer sequences

\begin{tabular}{ll}
\hline Primer & Sequences \\
\hline SEO_Fwd & CGT ATC GCC TCC CTC GCG CCA TCA G [MID sequence] \\
SEO_Rev & TGG AAT CTT TGC CCA GAT GG \\
& CTA TGC GCC TTG CCA GCC CGC TCA G [MID sequence] \\
& ACA ACC AAT CCC GTG GC TAC
\end{tabular}

MID, NGS identifier sequence; NGS, next generation sequencing.

Supplementary Table S2. Characterization of AAV vector preparations

\begin{tabular}{lccc}
\hline Vectors & $\begin{array}{c}\text { Genomic titer } \\
\left(\times 10^{8} / \mu L\right)\end{array}$ & $\begin{array}{c}\text { Capsid titer } \\
\left(\times 10^{9} / \mu L\right)\end{array}$ & $\begin{array}{c}\text { Capsid-to-genomic } \\
\text { ratio }\end{array}$ \\
\hline AAV2 & $3.09 \pm 2.93$ & $5.96 \pm 6.71$ & $16.37 \pm 6.18$ \\
AAV- $N_{\mathrm{EC}}$ & $2.90 \pm 2.33$ & $2.91 \pm 2.71$ & $9.28 \pm 1.87$ \\
AAV-V & $2.79 \pm 3.00$ & $5.96 \pm 7.46$ & $16.61 \pm 8.88$
\end{tabular}

Indicated viral vectors were produced at least three times independently. Genomic titer (vector genome containing particles per microliter), capsid titer (intact capsids per microliter), and packaging efficiency were determined. Packaging efficiency is defined as the ratio of capsid titerto-genomic titer. Wild-type phenotype: below $50^{\mathrm{S} 1}$ Values are mean of three independent experiments \pm standard deviation.

AAV2, adeno-associated viral serotype 2; EC, endothelial cells.

\section{SUPPLEMENTARY REFERENCE}

S1. Kern A, Schmidt K, Leder C, et al. Identification of a heparin-binding motif on adeno-associated virus type 2 capsids. J Virol 2003;77:11072-11081. 


\section{AUTHOR QUERY FOR HUM-2019-027-VER9-ZHANG 1P}

AU1: Please note that gene symbols in any article should be formatted as per the gene nomenclature. Thus, please make sure that gene symbols, if any in this article, are italicized.

AU2: Please identify (highlight or circle) all authors' surnames for accurate indexing citations.

AU3: Please confirm the correctness of authors' affiliations.

AU4: Please mention division or department, if any, in author's affiliations 1-8 and 11.

AU5: The Publisher requests for readability that no paragraph exceeds 15 typeset lines. Please check for long paragraphs and divide where needed.

AU6: Please fix the expansion of the acronym "AAV": "adeno-associated viruses" or "adeno-associated viral"?

AU7: Please expand "pLG."

AU8: Please expand "CLO."

AU9: Supplementary Figure S2 citation is not in sequential order in the text. Please check.

AU10: Please expand "MEF."

AU11: Please mention the appropriate figure number in "figure."

AU12: Please define "SIGYPLP."

AU13: Please expand "RXXR."

AU14: Please provide the grant numbers for funding agency(ies)/institution(s).

AU15: Please expand "GAPDH."

AU16: Please define "DAPI." 